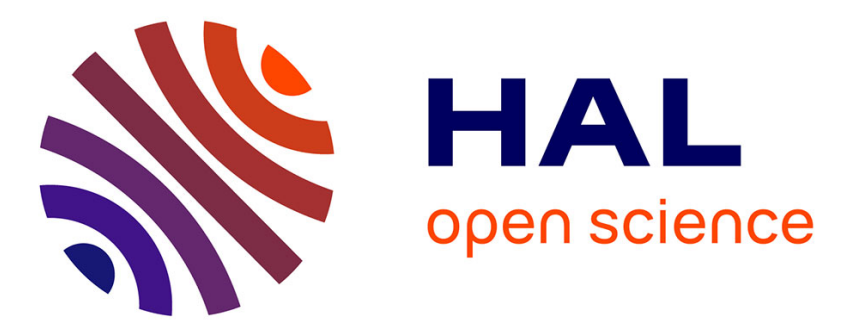

\title{
A new accurate ground-state potential energy surface of ethylene and predictions for rotational and vibrational energy levels.
}

Thibault Delahaye, Andrei Nikitin, Michaël Rey, Peter G. Szalay, Vladimir G. Tyuterev

\section{To cite this version:}

Thibault Delahaye, Andrei Nikitin, Michaël Rey, Peter G. Szalay, Vladimir G. Tyuterev. A new accurate ground-state potential energy surface of ethylene and predictions for rotational and vibrational energy levels.. Journal of Chemical Physics, 2014, 141, pp.104301. 10.1063/1.4894419 . hal-01214173

\section{HAL Id: hal-01214173 \\ https://hal.science/hal-01214173}

Submitted on 12 Oct 2015

HAL is a multi-disciplinary open access archive for the deposit and dissemination of scientific research documents, whether they are published or not. The documents may come from teaching and research institutions in France or abroad, or from public or private research centers.
L'archive ouverte pluridisciplinaire HAL, est destinée au dépôt et à la diffusion de documents scientifiques de niveau recherche, publiés ou non, émanant des établissements d'enseignement et de recherche français ou étrangers, des laboratoires publics ou privés. 


\title{
A new accurate ground-state potential energy surface of ethylene and predictions for rotational and vibrational energy levels
}

\author{
Thibault Delahaye, ${ }^{1}$, a) Andrei Nikitin, ${ }^{2,3}$ Michaël Rey, ${ }^{1, b)}$ Péter G. Szalay, ${ }^{4}$ and Vladimir G. Tyuterev ${ }^{1}$ \\ ${ }^{1)}$ Groupe de Spectrométrie Moléculaire et Atmosphérique, UMR CNRS 7331, BP 1039, F-51687, Reims Cedex 2, \\ France \\ ${ }^{2)}$ Laboratory of Theoretical Spectroscopy, Institute of Atmospheric Optics, Russian Academy of Sciences, \\ 634055 Tomsk, Russia \\ 3) Quamer, State University of Tomsk, Russia \\ ${ }^{4)}$ Institute of Chemistry, Eötvös Loránd University, P.O. Box 32, H-1518 Budapest, Hungary
}

(Dated: October 10, 2015)

In this paper we report a new ground state potential energy surface for ethylene (ethene) $\mathrm{C}_{2} \mathrm{H}_{4}$ obtained from extended $a b$ initio calculations. The coupled-cluster approach with the perturbative inclusion of the connected triple excitations $\operatorname{CCSD}(\mathrm{T})$ and correlation consistent polarized valence basis set cc-pVQZ was employed for computations of electronic ground state energies. The fit of the surface included 82542 nuclear configurations using sixth order expansion in curvilinear symmetry-adapted coordinates involving 2236 parameters. A good convergence for variationally-computed vibrational levels of the $\mathrm{C}_{2} \mathrm{H}_{4}$ molecule was obtained with a RMS(Obs. - Calc.) deviation of $2.7 \mathrm{~cm}^{-1}$ for fundamental bands centers and $5.9 \mathrm{~cm}^{-1}$ for vibrational bands up to $7800 \mathrm{~cm}^{-1}$. Large scale vibrational and rotational calculations for ${ }^{12} \mathrm{C}_{2} \mathrm{H}_{4},{ }^{13} \mathrm{C}_{2} \mathrm{H}_{4}$ and ${ }^{12} \mathrm{C}_{2} \mathrm{D}_{4}$ isotopologues were performed using this new surface. Energy levels for $J=20 \mathrm{up} \mathrm{to} 6000 \mathrm{~cm}^{-1}$ are in a good agreement with observations. This represents a considerable improvement with respect to available global predictions of vibrational levels of ${ }^{13} \mathrm{C}_{2} \mathrm{H}_{4}$ and ${ }^{12} \mathrm{C}_{2} \mathrm{D}_{4}$ and rovibrational levels of ${ }^{12} \mathrm{C}_{2} \mathrm{H}_{4}$.

Copyright 2014 American Institute of Physics. This article may be downloaded for personal use only. Any other use requires prior permission of the author and the American Institute of Physics. The following article appeared in J. Chem. Phys. 141, 104301 (2014) and may be found at http://scitation.aip.org/content/ aip/journal/jcp/141/10/10.1063/1.4894419.

\section{INTRODUCTION}

Polyatomic molecules such as methane $\mathrm{CH}_{4}$, acetylene $\mathrm{C}_{2} \mathrm{H}_{2}$ and ethylene (ethene) $\mathrm{C}_{2} \mathrm{H}_{4}$ "carbon chains" are of considerable interest for modeling of planetary atmospheres and other astrophysical applications. They present strong absorption in the overtone range usually corresponding to excitation of the $\mathrm{CH}$ bonds, so that these small hydrocarbons dominate the opacity of some brown dwarfs, exoplanets and asymptotic-giant-branch (AGB) stars and play a primary role in the physical chemistry of their outer atmospheres ${ }^{1}$. Thus, the emission of AGBs is partly affected by ethylene which has been detected in the outer shell of IRC+1021641 and IRL61842 where it is proposed that methane combustion may generate $\mathrm{C}_{2} \mathrm{H}_{4}$ and $\mathrm{HCN}$. Knowledge of rovibrational transitions of hydrocarbons is of primary importance in many fields, as for example for identifying the chemical composition of Titan atmosphere ${ }^{2}$ but remains a formidable challenge for the theory and spectral analysis ${ }^{3}$.

Several databases aim at collecting the corresponding data ${ }^{4-6}$ but the information about $\mathrm{C}_{2} \mathrm{H}_{4}$ spectrum present in databases remains limited, only some spectral ranges around 1000 and $3000 \mathrm{~cm}^{-1}$ being available.

\footnotetext{
a) Electronic mail: thibault.delahaye@univ-reims.fr

b) Electronic mail: michael.rey@univ-reims.fr
}

Many recent studies on vibrational fundamental ${ }^{7-11}$ and combination $^{8,12}$ band analysis tend to increase these data up to $6000 \mathrm{~cm}^{-1}$.

Both the development of high-quality $a b$ initio potential energy surface (PES) and dipole moment surface (DMS) and improvement in computational codes solving the rovibrational Schrödinger equation have been recently achieved (see for example Refs. 13-30 and references therein, the list being not exhaustive). All these made the calculation of quantitatively accurate $a b$ initio rovibrational spectra up to five atomic molecules possible. These $a b$ initio predictions of the PES and molecular properties for small molecules help resolving many issues related to the analysis of the spectra ${ }^{31}$.

Theoretical predictions proved to be very useful for analysis of experimental spectra at high-energy ranges. Obviously a construction of twelve-dimensional surfaces for six-atomic molecules is a considerable challenge for the theory. Avila and Carrington ${ }^{32}$ reported $J=0$ vibrational energies of almost 130 states of ${ }^{12} \mathrm{C}_{2} \mathrm{H}_{4}$ from a CCSD(T)/cc-pVTZ modified force field, initially computed by Martin et al. ${ }^{33}$ in a polynomial representation, and extended by using Morse variables for the four $\mathrm{C}-\mathrm{H}$ stretch coordinates. Thereafter Carter et al. ${ }^{34}$ used this modified potential to perform rovibrational variational calculations with the Multimode code, using a basis with typical size of VCI $A_{g}$ symmetry block of 24048 for accurate determination of $J=0,1,2$ energies and a reduced basis (size of $A_{g}$ symmetry block $=2654$ ) for 
more qualitative predictions up to $J=40$. Both studies showed a rather good description of fundamentals and to a less extent of first overtones and combination bands. These authors concluded that their calculations were mostly limited by the accuracy of the potential and pointed out the need of a new surface, obtained at a higher level of the $a b$ initio theory, a larger basis and advanced fitting methods.

In this paper, we report a new PES for ethylene which was calculated using augmented coupled cluster CCSD(T) method and correlation consistent basis set ccpVQZ. In order to validate the accuracy of the surface, we also present vibration and rotation-vibration predictions using normal mode variational techniques. For a polyatomic molecule in normal coordinates the vibrationrotation Hamiltonian has been formulated in its most compact form by Watson ${ }^{35}$ and is referred to as EckartWatson Hamiltonian (EWH) in the following. In case of nonlinear molecules as ethylene the EWH is given by

$$
\begin{aligned}
H(\boldsymbol{p}, \boldsymbol{q}, \boldsymbol{J})= & \frac{1}{2} \sum_{k=1}^{3 N-6} \omega_{k} p_{k}^{2}+U(\boldsymbol{q})+U_{W}(\boldsymbol{q}) \\
& +\frac{1}{2} \sum_{\alpha \beta}\left(J_{\alpha}-\pi_{\alpha}\right) \mu_{\alpha \beta}\left(J_{\beta}-\pi_{\beta}\right),
\end{aligned}
$$

where $\alpha, \beta=x, y, z, J_{\alpha}$ and $\pi_{\alpha}$ are molecular frame components of the total and vibrational angular momentum, respectively, and $\boldsymbol{\mu}$ is the reciprocal inertia tensor. $U(\mathbf{q})$ is the potential function and $U_{W}(\mathbf{q})=-\frac{1}{8} \sum \mu_{\alpha \alpha}$ is a small mass-dependent contribution introduced by Watson that is a purely quantum mechanical term of kinetic origin $^{35}$. In this representation the quantum nuclear motion in the twelveth-dimensional vibrational space is described by the set of rectilinear normal coordinates $\mathbf{q}$. Computations for solving the rovibrational Schrödinger equation using this EWH were performed with our recent in-house code Tensor. Besides providing all necessary transformations for a systematic symmetry-adapted development of the complete normal-mode Hamiltonian expansion, this computational code implements variational procedure and reduction-truncation techniques for rovibrational spectrum predictions.

The paper is structured as follows. We give in Sections II and III computational details about the new $a b$ initio PES for electronic structure calculations and for surface fitting procedures. Sections IV, V and VI present succinct description of procedures implemented in the TENSOR code, vibrational and rovibrational energies of ${ }^{12} \mathrm{C}_{2} \mathrm{H}_{4}$ and isotopologues ${ }^{12} \mathrm{C}_{2} \mathrm{D}_{4}$ and ${ }^{13} \mathrm{C}_{2} \mathrm{H}_{4}$ up to $J=20$. Comparisons with experimental data and recent works are also reported. A brief summary and conclusions are given in Section VII.

\section{ELECTRONIC STRUCTURE COMPUTATIONS}

Accurate calculations of vibrational and rotational energy levels from a PES require combining high level $a b$ initio methods with sufficiently large basis sets in electronic structure calculations. For this purpose, we used the well-known coupled cluster approach including single and double excitations ${ }^{36}$ and the perturbative treatment of triple excitations, usually denoted as $\operatorname{CCSD}(\mathrm{T})$ method $^{37}$. Calculations were carried out using well established Dunning's correlation consistent basis sets cc-pVQZ ${ }^{38}$. The MolPRO program package version $2009.1^{39}$ was used to perform all the ab initio calculations of electronic ground state energies. Most of the calculations were using the regional "Romeo" multiprocessor computer (Reims), "IDRIS" computer centre of CNRS in Orsay and "JADE" cluster at CINES computer centre in Montpellier. As a first step for the PES construction, a set of ground state electronic energies was calculated with cc-pVQZ basis set on a grid of 82542 nuclear configurations described in the following Section IIB. Together, these calculations took about 30000 hours (CPU time). Density distribution of included geometrical configurations is given in Figure 1 with a maximum number of configurations near $5000-7000 \mathrm{~cm}^{-1}$ but a significant number of points extends up to $12000-13000 \mathrm{~cm}^{-1}$.

\section{A. Equilibrium structure}

A full PES suitable for quantitatively accurate prediction of highly excited vibration-rotation states of ethylene requires accurate determination of the equilibrium structure of the molecule, otherwise the precision of rotational levels dramatically decreases with increasing $J$ values. Due to the $D_{2 h}$ symmetry of the ethylene molecule, the equilibrium geometry is defined by only three parameters, $r_{e}(\mathrm{C}-\mathrm{H}), r_{e}(\mathrm{C}-\mathrm{C})$ and $\alpha_{e}(\mathrm{HCC})$. Thereafter, these parameters are referred to as $r_{H_{e}}, r_{C_{e}}$ and $\alpha_{e}$. As the rotational constants depend on $\mathrm{C}_{2} \mathrm{H}_{4}$ equilibrium structure, these three parameters are of primary importance for accurate description of rotational spectra.

Previous works considered equilibrium geometry based on $a b$ initio geometry optimization at $\operatorname{CCSD}(\mathrm{T}) / \mathrm{cc}-$ pVTZ level of the theory. Nevertheless it seems clear that even a $\operatorname{CCSD}(\mathrm{T}) / \mathrm{cc}-\mathrm{pVQZ}$ optimized geometry cannot reach a sufficient precision for rotational predictions. Some results for the equilibrium geometry optimization obtained using different level of the theory are summarized in Table I. Increasing the basis set dimension does not lead necessarily to a smooth convergence for equilibrium values. However, a general trend can be observed. Both cc-pVQZ and cc-pV5Z valence basis sets result in somewhat larger $r_{H_{e}}$ and $r_{C_{e}}$ values and smaller $\alpha_{e}$ angle. On the other hand, including larger core valence basis sets cc-pCVQZ and cc-pCV5Z gives shorter bond lengths values and wider $\alpha_{e}$ angle. Including augmented basis with diffuse functions results in a smaller effect on 
Table I. Equilibrium geometry parameters of $\mathrm{C}_{2} \mathrm{H}_{4}$ optimized using $\mathrm{CCSD}(\mathrm{T})$ method with different basis sets and corresponding ground state rotational $J=1$ levels.

\begin{tabular}{|c|c|c|c|c|c|c|c|c|}
\hline Basis set & $\#^{a}$ & $\mathrm{r}_{e}(\mathrm{C}-\mathrm{H})(\AA)$ & $\mathrm{r}_{e}(\mathrm{C}-\mathrm{C})(\AA)$ & $\alpha_{e}(\mathrm{HCC})\left({ }^{\circ}\right)$ & $\begin{array}{l}\text { Calculated }^{b} \\
\mathrm{E}_{J=1, k_{a}=0, k_{c}=1}\end{array}$ & $\begin{array}{l}\text { Calculated }^{b} \\
\mathrm{E}_{J=1, k_{a}=1, k_{c}=1}\end{array}$ & $\begin{array}{l}\text { Calculated }^{b} \\
\mathrm{E}_{J=1, k_{a}=1, k_{c}=0}\end{array}$ & $\Delta^{c}\left(\mathrm{~cm}^{-1}\right)$ \\
\hline cc-pVQZ & 230 & 1.082353 & 1.334280 & 121.437799 & 1.82015 & 5.67651 & 5.84837 & 0.01453 \\
\hline cc-pAV5Z & 574 & 1.082217 & 1.333849 & 121.423498 & 1.82135 & 5.67672 & 5.84881 & 0.01404 \\
\hline cc-pV5Z & 402 & 1.082091 & 1.333714 & 121.426600 & 1.82168 & 5.67672 & 5.85044 & 0.01273 \\
\hline cc-pCV5Z & 510 & 1.080604 & 1.330585 & 121.435877 & 1.82927 & 5.69586 & 5.86889 & 0.00271 \\
\hline cc-pACVQZ & 402 & 1.081178 & 1.331679 & 121.443120 & 1.82643 & 5.69026 & 5.86291 & 0.00254 \\
\hline cc-pACV5Z & 682 & 1.080729 & 1.330734 & 121.432973 & 1.82889 & 5.69426 & 5.86728 & 0.00140 \\
\hline cc-pCVQZ & 288 & 1.080918 & 1.331200 & 121.447357 & 1.82758 & 5.69353 & 5.86631 & 0.00113 \\
\hline Our Empiric ${ }^{d}$ & - & 1.080565 & 1.330898 & 121.401760 & 1.82909 & 5.69253 & 5.86561 & 0.00005 \\
\hline
\end{tabular}

a Number of contracted functions.

${ }^{b}$ Calculated energy levels of $\mathrm{C}_{2} \mathrm{H}_{4}$ in $\mathrm{cm}^{-1}$ using atomic masses and our PES in normal coordinates with corresponding equilibrium configuration (see Section II A).

c $\Delta$ is a RMS(obs.-calc.) deviation for the $J=1$ levels with respect to Ref. 8 .

${ }^{d}$ Our optimized value obtained by empirical procedure described in section II A.

parameters values but obviously tends to slightly relax bond lengths and $\alpha_{e}(\mathrm{HCH})$ angle. In terms of bond lengths and angle a certain regularity can be seen: irrespective of core function or augmentation, the bond length and angle get smaller when going from QZ to $5 \mathrm{Z}$. Also adding core functions or augmentation, the results get smaller irrespective whether we use $5 \mathrm{Z}$ or QZ basis. Nevertheless, the $\Delta_{\text {(Obs.-Calc) }}$ RMS deviation for $J=1$ levels was not as accurate as in our previous study for the methane molecule ${ }^{26}$. In order to improve the equilibrium geometry for the purpose of variational spectra calculations, we applied a simple iterative algorithm for an optimization of $r_{H_{e}}, r_{C_{e}}$ and $\alpha_{e}$ parameters using observed ground state $J=1$ levels of $\mathrm{C}_{2} \mathrm{H}_{4}$ which guided iterative corrections to $A_{e}, B_{e}$ and $C_{e}$. This simple procedure leads to an improvement by more than one order of magnitude for the ground state $E_{J=1}$ rotation energies compared to purely ab initio optimized geometries. The main idea behind this optimization procedure is based

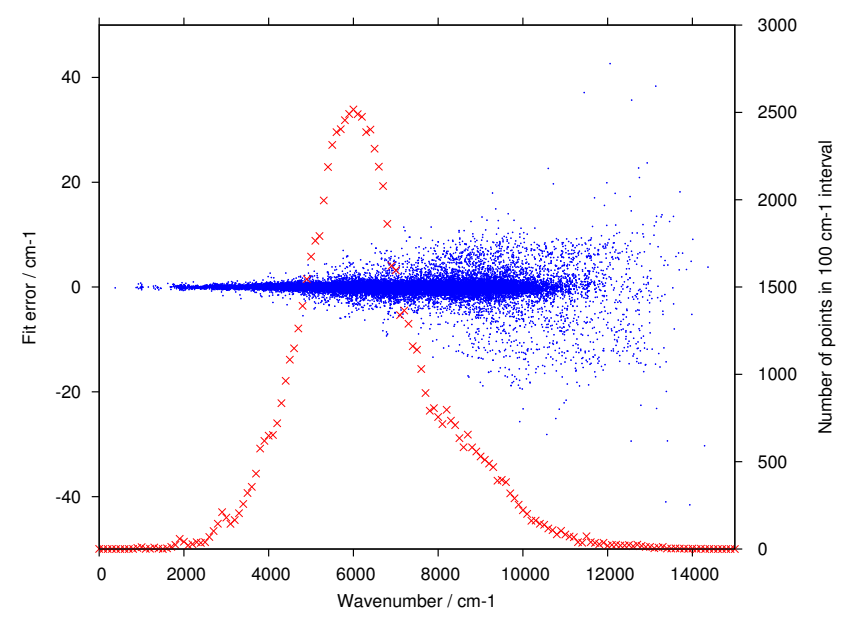

Figure 1. Density distribution of calculated $a b$ initio points (red cross on the right-hand scale) and errors of the PES fit to $a b$ initio electronic energies (blue dots on the left-hand side scale). (For interpretation of the references to color in this figure, the reader is referred to the web version of this article.) on the assumption that PESs computed with basis sets listed in Table I have very similar shapes at least near the bottom of the potential and that the major contribution to rotational levels comes from relatively small changes in the equilibrium geometry parameters. As our best $a b$ initio equilibrium was very close to the final empirically corrected value (variation of $\simeq 0.0004 \AA$ for bonds and 0.04 Degrees for the angle, Table I) only "fine tuning" was necessary: $A_{e}, B_{e}$ and $C_{e}$ were adjusted without changing the PES expansion terms. We have checked that in our case these tiny changes in equilibrium corrections result in quite small changes $\simeq 0.01-0.1 \mathrm{~cm}^{-1}$ in fundamental vibration energies that is less than the accuracy of the $a b$ initio PES. Extrapolations to higher rotational levels discussed in Section VI confirm that this empirically optimized equilibrium geometry is appropriate for rotational calculations using atomic masses and the PES shape determined at $\operatorname{CCSD}(\mathrm{T}) / \mathrm{cc}-\mathrm{pVQZ}$ level of the $a b$ initio theory. Our procedure is quite similar in spirit to the method for an empirical optimization for equilibrium geometries of Pawlowski et $a l^{40}$. The main difference is that Pawlowski et al..$^{40}$ used second order perturbation theory for the vibration-rotation terms limited by including quadratic and cubic force constants only, whereas our approach is fully variational accounting for higher order terms of the PES expansion. It is instructive to compare our empirically optimized equilibrium configuration with Pawlowski et al. results who reported the following values: $r_{H_{e}}=1.08068( \pm 0.0006) \AA$, $r_{C_{e}}=1.33074( \pm 0.0008) \AA, \alpha_{e}=121.4( \pm 0.24)$ Degrees [Tables XIII, XIV of Ref. 40]. The differences with our optimized values are $0.0001 \AA, 0.0002 \AA$ and 0.0018 Degrees, respectively, which are below the uncertainties of Pawlowski et al. determinations.

\section{B. Sampling of ab initio points in the nuclear configuration space}

Twelve internal symmetry-adapted coordinates are necessary to describe the PES and the vibrational motion of the $\mathrm{C}_{2} \mathrm{H}_{4}$ molecule. To parametrize all possible nuclear geometries of the system, it is convenient to use 
polar curvilinear valence coordinates because they are internally built in ab initio programs like MoLPRo. The internal polar coordinates are defined in a standard way via four vectors $\left\{\vec{r}_{i}\right\} \quad(i=1,2,3,4)$ linking the four $\mathrm{H}$ atoms to the closest $\mathrm{C}$ atom and $\vec{r}_{5}$ linking the two $\mathrm{C}$ atoms together. The following coordinates were chosen as independent ones: four $\mathrm{CH}$ bond lengths $\left\{r_{1}, r_{2}, r_{3}, r_{4}\right\}$, one $\mathrm{CC}$ bond length $\left\{r_{5}\right\}$ and four $\mathrm{CCH}$ bond angles $\left\{q_{1}, q_{2}, q_{3}, q_{4}\right\}$. In addition three independent torsion angles need to be defined. Though it is more convenient to deal with four torsion coordinates corresponding to the two trans- $\left\{t_{1564}, t_{2563}\right\}$ and two cis- $\left\{t_{1563}, t_{2564}\right\} \mathrm{H}-$ $\mathrm{C}-\mathrm{C}-\mathrm{H}$ torsion angles (with respect to atom numbering in Figure 2), and referred as $\left\{t_{1}, t_{2}, t_{3}, t_{4}\right\}$, respectively, only three combinations of $\left\{t_{i}\right\}$ are independent.

We follow the technique described in Refs. 18 and 26 to derive a symmetry-adapted grid of points in the coordinate space suitable for a reliable determination of PES parameters. This approach allows a determination of an optimal set of geometric nuclear configurations sufficient for a construction of the force field up to a certain order of expansion. The twelve-dimensional PES was constructed in a five step process:

(i) One dimensional curves corresponding to each of twelve symmetrized coordinates were constructed first. These one-dimensional curves were interpolated using the sixth order power series expansions. Then the values of the symmetrized coordinates where the PES should take values 0, 1000, 2500, $4000,7000,12000,18000,25000 \mathrm{~cm}^{-1}$ were found.

(ii) A full set of 17000 symmetry-adapted terms for the $D_{2 h}$ group up to the $8^{t h}$ order expansion was constructed. Every term was represented as the sum of products of symmetry-adapted coordinates $S_{i}$, defined by Eqs 2-3 of the next Section III.

Table II. Harmonic frequencies of ${ }^{12} \mathrm{C}_{2} \mathrm{H}_{4}$.

\begin{tabular}{|c|c|c|}
\hline & This work & Emp. $^{a}$ \\
\hline$\omega_{1} \quad A_{g}$ S. CH stretch & 3156.84 & 3156.2 \\
\hline$\omega_{2} \quad A_{g}$ CC stretch & 1672.57 & 1656.4 \\
\hline$\omega_{3} \quad A_{g}$ S. HCH bend & 1369.38 & 1372.3 \\
\hline$\omega_{4} \quad A_{u} \mathrm{H}_{2} \mathrm{C}-\mathrm{CH}_{2}$ twist & 1050.81 & 1044.6 \\
\hline$B_{2 g}$ trans $\mathrm{CH}$ stretch & 3222.89 & 3206.9 \\
\hline$B_{2 g}$ A. $\mathrm{HCH}$ wag & 1246.76 & 1248.5 \\
\hline$B_{2 u}$ S. out of plane & 966.39 & 968.3 \\
\hline$B_{3 g}$ A. out of plane & 950.19 & 960.3 \\
\hline$\omega_{9} \quad B_{3 u}$ cis $\mathrm{CH}$ stretch & 3248.71 & 3239.1 \\
\hline$\omega_{10} B_{3 u}$ S. HCH wag & 824.97 & 844.1 \\
\hline$\omega_{11} B_{1 u}$ A. CH stretch & 3140.91 & $3147.0^{41}$ \\
\hline$\omega_{12} \quad B_{1 u}$ A. HCH bend & 1478.48 & 1471.8 \\
\hline
\end{tabular}

All values in $\mathrm{cm}^{-1}$. S. and A. stand for "Symmetric" and "Antisymmetric".

${ }^{a}$ Estimations from empirical model of Ref. 42 if no other reference is given. (iii) A part of terms corresponding to powers $>6$ of the radial $\mathrm{CH}$ coordinates were suppressed from the further calculations in order to keep reasonable number of points. This is because preliminary estimations showed that more angular points were required for converging nuclear motion calculations.

(iv) All points for the grid in the $\left\{S_{i}\right\}$ nuclear configuration space necessary for finite difference method determination of all above mentioned contributions were found. We followed the method previously applied for the methane molecule ${ }^{26}$.

(v) Coincident configurations $\left\{S_{i}\right\}$ after permutations of identical atoms were deleted from the final list of grid points $\left\{S_{i}\right\}$.

\section{FITTING OF PES EXPANSION PARAMETERS TO AB INITIO ELECTRONIC ENERGIES}

The total number of 82542 points for the optimal grid in the nuclear configuration space was built as described in Section IIB. In order to build the PES expansion we define then suitable elementary analytical functions of the stretching $(r)$ and angular $(q$ and $t)$ coordinates. The following elementary functions are used:

$$
\begin{aligned}
\phi\left(r_{i}\right) & =1-\exp \left[-a\left(r_{i}-r_{e}\right)\right] \\
\phi\left(q_{i}\right) & =q_{i}-q_{e} \\
\phi\left(t_{i}\right) & =t_{i}-t_{e}
\end{aligned}
$$

where $a=1.9$. This value of the $a$ parameter ensures that the second order term of the potential expansion provides a reliable representation for the one dimensional stretching cut. Terms of higher orders result in relatively small corrections. Initially the set of equilibrium nuclear configuration parameters $\left\{r_{e}, q_{e}, t_{e}\right\}$ was taken at the same level of $a b$ initio calculations as the PES calculations, $i e$ at the $\operatorname{CCSD}(\mathrm{T}) / \mathrm{cc}-\mathrm{pVQZ}$ level. This was necessary to minimize the deviation beetween $a b$ initio points and the analytical representation during the fitting procedure. Thereafter, for the Hamiltonian development, the PES was expanded around the equilibrium that ensures the best representation of rotational levels (see empirical equilibrium described in Section II A). This approximation implies that rotational levels are mainly determined by rotational constants (and therefore by the equilibrium geometry), and vibrational levels by the force field shape. With this technique we keep the force field and translate it to a better equilibrium representation for rotational levels. Such an assumption is only valid if the deviation of empirically optimized equilibrium configuration from the $a b$ initio one is very small. A comparison given in Table I certifies that this is indeed the case.

For the fitting procedure, one has to choose properly the molecular axis frame. Actually, there are two commonly used conventions, namely the IUPAC and Herzberg axes conventions (see Fig. 2), but a third one, 


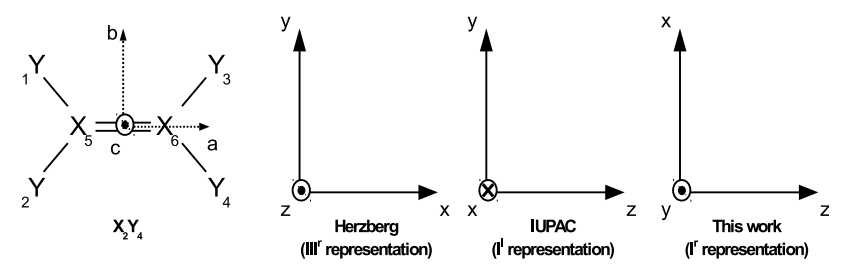

Figure 2. Molecular axis frame conventions for $\mathrm{X}_{2} \mathrm{Y}_{4}$ systems.

the $I^{r}$ representation is generally used for the calculation of rotational levels ${ }^{8}$. In this work we have adopted the molecular fixed Eckart axis embedding with the $I^{r}$ representation for the rovibrational calculations. The abovedefined elementary functions of Eq. 2 were used to build twelve symmetrized $S_{i}^{(\Gamma)}$ coordinates which are nonlinear with respect to the stretching displacements,

$$
\begin{aligned}
S_{1}^{\left(A_{g}\right)} & =\left(\phi\left(r_{1}\right)+\phi\left(r_{2}\right)+\phi\left(r_{3}\right)+\phi\left(r_{4}\right)\right) / 2 \\
S_{2}^{\left(A_{g}\right)} & =\phi\left(r_{5}\right) \\
S_{3}^{\left(A_{g}\right)} & =\left(\phi\left(q_{1}\right)+\phi\left(q_{2}\right)+\phi\left(q_{3}\right)+\phi\left(q_{4}\right)\right) / 2 \\
S_{4}^{\left(A_{u}\right)} & \left.=\left(\phi\left(t_{3}\right)+\phi\left(t_{4}\right)\right)\right) / \sqrt{2} \\
S_{5}^{\left(B_{2 g}\right)} & =\left(-\phi\left(r_{1}\right)+\phi\left(r_{2}\right)+\phi\left(r_{3}\right)-\phi\left(r_{4}\right)\right) / 2 \\
S_{6}^{\left(B_{2 g}\right)} & =\left(-\phi\left(q_{1}\right)+\phi\left(q_{2}\right)+\phi\left(q_{3}\right)-\phi\left(q_{4}\right)\right) / 2 \\
S_{7}^{\left(B_{2 u}\right)} & \left.=\left(\phi\left(t_{2}\right)-\phi\left(t_{1}\right)\right)\right) / \sqrt{2} \\
S_{8}^{\left(B_{3 g}\right)} & \left.=\left(\phi\left(t_{3}\right)-\phi\left(t_{4}\right)\right)\right) / \sqrt{2} \\
S_{9}^{\left(B_{3 u}\right)} & =\left(\phi\left(r_{1}\right)-\phi\left(r_{2}\right)+\phi\left(r_{3}\right)-\phi\left(r_{4}\right)\right) / 2 \\
S_{10}^{\left(B_{3 u}\right)} & =\left(\phi\left(q_{1}\right)-\phi\left(q_{2}\right)+\phi\left(q_{3}\right)-\phi\left(q_{4}\right)\right) / 2 \\
S_{11}^{\left(B_{1 u}\right)} & =\left(-\phi\left(r_{1}\right)-\phi\left(r_{2}\right)+\phi\left(r_{3}\right)+\phi\left(r_{4}\right)\right) / 2 \\
S_{12}^{\left(B_{1 u}\right)} & =\left(-\phi\left(q_{1}\right)-\phi\left(q_{2}\right)+\phi\left(q_{3}\right)+\phi\left(q_{4}\right)\right) / 2 .
\end{aligned}
$$

The upper case indices correspond to the irreducible representations (irreps) of the $D_{2 h}$ point group. In order to determine the 12D potential expansion terms (each of the $A_{g}$ total symmetric type), formed from the symmetrised coordinates $S_{i}$, we applied a two-step procedure. The first step corresponds to the construction of the symmetrised powers of $S_{i}$ and the second step to the coupling

Table III. Statistics for the PES fit to $a b$ initio electronic energies.

\begin{tabular}{lcl}
\hline $\mathrm{E}_{\max }{ }^{a}$ & number of points & $\mathrm{rms}^{b}$ \\
\hline 5000 & 14625 & 0.242 \\
10000 & 80383 & 0.939 \\
15000 & 82542 & 1.369 \\
\hline
\end{tabular}

${ }^{a}$ Threshold for electronic energies above equilibrium, in $\mathrm{cm}^{-1}$.

${ }^{b}$ rms deviation between $a b$ initio points and analytical PES representation, in $\mathrm{cm}^{-1}$. of the symmetrised powers of different symmetrised coordinates in irreducible balanced trees according to the algorithm of Ref. 43. The same technique has been already used for the construction of the methane $\mathrm{PES}^{26}$. A set of all possible trees of the totally symmetric $A_{g}$ representation gives a final set of the $12 \mathrm{D}$ expansion terms. The potential function was finally developed in power series of symmetry-adapted coordinates defined in Eq. (3)

$$
V\left(r_{i}, q_{j}, t_{l}\right)=\sum_{n} K_{n} R_{n}^{p}\left(r_{i}, q_{j}, t_{l}\right)
$$

where $i, j, l \in\{1,2,3,4\}$ and

$$
\begin{aligned}
R_{n}^{p}\left(r_{i}, q_{j}, t_{l}\right) & =\left(S_{1_{A_{g}}}^{p_{1}} S_{2_{A_{g}}}^{p_{2}} S_{3_{A_{g}}}^{p_{3}} S_{4_{A_{u}}}^{p_{4}} S_{5_{B_{2} g}}^{p_{5}} S_{6_{B_{2 g}}}^{p_{6}} S_{7_{B_{2 u}}}^{p_{7}}\right. \\
& \left.\times S_{8_{B_{3 g}}}^{p_{8}} S_{9_{B_{3 u}}}^{p_{9}} S_{1_{B_{3 u}}}^{p_{10}} S_{1_{B_{1 u}}}^{p_{11}} S_{1_{B_{1 u}}}^{p_{12}}\right)^{A_{g}}
\end{aligned}
$$

and $p=\sum_{m=1}^{12} p_{m}$. Here $n$ is the string of indices defined by Eq. (5). The maximum power $p_{\max }$ in the truncated expansion (4) is referred to as the order of the PES. We fitted our $a b$ initio potential energies using the analytical symmetry-adapted representation (2)-(4). A similar weight function (depending on energy $E$ in $\mathrm{cm}^{-1}$ )

$$
w(E)=\frac{\tanh (-0.0005(E-9000)+1.002002002)}{2.002002002}
$$

to that employed by Schwenke and Partridge in Ref. 44 was used in the weighted fit of PES parameters to $a b$ initio electronic energies. This form of weighting function minimizes the relative error, by de-emphasizing energies above $V_{\text {top }}=9000 \mathrm{~cm}^{-1}$ and keeping a maximum weight for the lowest energy points. More details can be found in Ref. 44. Among the 2654 parameters initially included in the surface fit at order $p_{\max }=6$, three linear parameters were suppressed to avoid a shift in the equilibrium configuration of the analytical PES representation. We found that 2236 parameters (of 2651 included) were statistically well determined in this fit on the entire grid of all $82542 a b$ initio points with deviations presented in Table III. We also give in Table II a comparison between our harmonic frequencies and those determined via an empirical data fitting model ${ }^{42}$. At the second step all poorly defined parameters were excluded. Figure 1 shows the distribution of the final fit of the $a b$ initio electronic energies using our analytical PES representation. The errors are quite small up to $\sim 10000 \mathrm{~cm}^{-1}$. A larger scatter of points above this range occurs because the weighting function (6) quickly de-emphasizes energies above this threshold $^{44}$. In order to further improve the fit, it would be necessary to include some higher order terms in the PES expansion (4). We plan to do this in a future work. 


\section{VARIATIONAL CALCULATION OF VIBRATION LEVELS OF ${ }^{12} \mathbf{C}_{2} \mathbf{H}_{4}$}

\section{A. Theoretical background}

For vibrational calculations, we use the EWH (1) and the variational approach implemented in our codes. It is well-known that exploiting molecular symmetry allows reducing the computational cost for solving the Schrödinger equation. One of the key features of our codes is a full use of symmetry properties throughout the calculation. Consequently, the Hamiltonian matrix is divided into eight blocks corresponding to the irreps of the symmetry point group $D_{2 h}$ of $\mathrm{C}_{2} \mathrm{H}_{4}$.

TEnsor program suite contains a set of procedures designed to reduce the dimension of matrices and to minimize the cost of the variational computation using a truncation-reduction techniques for the normal mode EWH symmetry-adapted expansion as described in Refs. 28 and 45. An advantage of this approach is to reduce the impacts of drawbacks of high-order Taylor series PES expansion at large distance (although high-order expansion is necessary for a good description of the shape of the potential) as recently proved to be efficient for the methane molecule ${ }^{45}$. A vibrational sub-space (VSS) procedure was also implemented in order to improve the convergence of high- $J$ rovibrational states ${ }^{45}$. This approach can be regarded as a kind of basis set compression technique for which somewhat other methods have been previously employed for molecules with fewer number of atoms ${ }^{13,16,46,47}$. Some related aspects have also been discussed in Refs. 48 and 49. In our case we use accurate vibrational eigenfunctions with an appropriate energy cut-off as a truncated basis set for vibration-rotation calculations. To summarize, the nuclear motion problem of $\mathrm{C}_{2} \mathrm{H}_{4}$ is solved in a four-step procedure:

(i) Reduction technique is applied to the vibrational part of the hamiltonian $H$ : we transform a $n^{\text {th }}$ order Taylor EWH $H^{(n)}(\boldsymbol{p}, \boldsymbol{q}, \boldsymbol{J})$ to a $m^{t h}$ order $(m<$ $n$ ) reduced Hamiltonian $H_{r e d}^{(n \rightarrow m)}(\boldsymbol{p}, \boldsymbol{q}, \boldsymbol{J})$ according to the method discussed in Refs. 28 and 45. Besides reducing the number of terms in the rovibrational expansion, making calculation of matrix elements much faster, this procedure has a fundamental impact on potential part by minimizing some impacts of Taylor artifacts, as discussed in the following Section IV B.

(ii) The vibrational primitive basis set $\left\{\Psi_{v}^{\left(C_{v}\right)}\right\}$ is built as a direct product of twelve harmonic oscillator (HO) normal mode functions,

$$
\left|\Psi_{v}^{\left(C_{v}\right)}\right\rangle=\left|\left(\Psi_{v_{1}}^{\left(A_{g}\right)} \times \Psi_{v_{2}}^{\left(A_{g}\right)} \times \ldots \times \Psi_{v_{12}}^{\left(B_{1 u}\right)}\right)^{\left(C_{v}\right)}\right\rangle,
$$

where $v_{1}, v_{2}, \ldots, v_{12}$ are the HO quantum numbers and $C_{v}$ is the vibrational symmetry type in the $D_{2 h}$ group, given by the multiplication rule :

$$
\begin{aligned}
C_{v}= & B_{2 g}{ }^{v_{5}+v_{6}} \times B_{3 g}{ }^{v_{8}} \times A_{u}^{v_{4}} \\
& \times B_{1 u}{ }^{v_{11}+v_{12}} \times B_{2 u^{v_{7}}} \times B_{3 u^{v_{9}+v_{10}}} .
\end{aligned}
$$

A cut-off criterion is imposed to select a limited set of primitive basis functions

$$
F_{\kappa}\left(v_{\max }\right)=\sum_{i} \kappa_{i} v_{i} \leq v_{\max }
$$

with $v_{i}=0, \ldots, v_{\max }$. A default option is a homogeneous cut-off with all $\kappa_{i}=1$. In the latter case we shall consider a concise notation $F_{\kappa=1}\left(v_{\max }\right) \equiv$ $F\left(v_{\max }\right)$. For $\mathrm{C}_{2} \mathrm{H}_{4}$, due to the large range covered by vibrational fundamentals (between 825 and 3100 $\mathrm{cm}^{-1}$ ), it is important to properly select a "pruning" scheme by defining appropriate $\kappa_{i}$ weight coefficients, and thus include a different number of stretching, bending and torsion basis functions.

(iii) The vibrational equation $H_{v} \Psi_{v}=E_{v} \Psi_{v}$ is variationally solved for a given basis set with the Hamiltonian model as described in Ref. 28. The corresponding eigensolutions $\left\{E_{v}, \Psi_{v}\right\}$ are stored in memory.

(iv) As the last step, the Hamiltonian is partitioned as $H=H_{v}+H_{r v}$ and the variational method is applied using anharmonic basis set $\left\{\Psi_{v}\right\}$ obtained at the previous step (iii) that permits improving the convergence of vibration-rotation calculations. By defining the cut-off for full vibrational energy $E_{\max }$, we make a basis set compression equivalent to a selection of an appropriate VSS spanned by variational eigensolutions of the step (iii) such that $E_{v}<E_{\max }$. This compact set of vibrational eigenfunctions is used to solve the full rovibrational eigen-problem for each $(J, C)$ block, where $J$ and symmetry labels $C=C_{v} \times C_{r}$ are used to assign levels. The VSS depends of course of the truncation $\left(v_{\max }\right)$ for the primitive HO normal mode basis set at the step (ii). At the step (iv) a use of the maximum primitive basis cut-off $\left(v_{\max }\right)$ for big rotational quantum numbers J could be too demanding. In this case we applied a further basis reduction: variational coefficients of wave function expansions were first optimized with the $\left(v_{\max }\right)$ cut-off and then transferred to the corresponding terms of a smaller basis set with $r<v_{\max }$. This truncationcompression scheme which has been discussed in detail in Ref. 28 proved to be efficient for calculations of methane spectra. The rovibrational energy levels are obtained by diagonalizing the resulting hamiltonian matrix expressed in the reduced vibrational eigenbasis. This permits handling much 
smaller matrices for the vibration-rotation eigensolutions and thus better converging variational spectra calculations.

In addition to drastically compressing the basis set size, the VSS procedure allows one to smoothly introduce empirical corrections for the band centers in rovibrational calculations. If we just replace $E_{v}$ by the observed values, all vibrational wavefunctions and all coupling matrix elements of $H_{r v}$ will not be affected by this change. They remain ab initio in origin, but vibration-rotation resonances are then better described at the step (iv). This model could provide a more realistic description of rovibrational perturbations in the spectra.

\section{B. Convergence properties and computational details}

Table IV summarizes the convergence tests involving the effect of the Hamiltonian expansions and reductions on vibrational levels. This includes the comparison of $H_{10}$ vibrational calculations with $H_{8}, H_{6}, H_{10 \rightarrow 8}, H_{10 \rightarrow 6}$, $H_{8 \rightarrow 6}$ and with the combined scheme $H_{10 \rightarrow 6}^{a 16 \rightarrow 8}$. The latter model was built from the reduced potential $\mathrm{V}_{\text {red }}^{(16 \rightarrow 8)}=$ $\mathrm{V}_{\text {red }}^{(10 \rightarrow 6)}+\mathrm{V}_{\text {red }}^{a(16 \rightarrow 8)}$ where the augmented angular contribution $\mathrm{V}_{\text {red }}^{a(16 \rightarrow 8)}$ is obtained as follows: first the PES was expanded in bending and torsion coordinates by fixing the stretching at the reference geometry, then the coupled angular terms from $11^{\text {th }}$ to $16^{\text {th }}$ orders were added and finally these supplementary terms were reduced to the $8^{\text {th }}$ order using $\left(a^{+}, a\right)$ representation.

One of major problem in the variational calculation of the rovibrational level is a possible presence of nonphysical PES features (spurious minima) in the range spanned by the wavefunctions of the considered states. In this study we did not experience server problems for the stretching degrees of freedom, but it was a challenging issue to describe correctly the angular behavior of the PES. Figure 3 shows bending and torsion PES cuts to illustrate an improvement of truncated Taylor expansion using the reduction schemes. This example shows that by choosing an appropriate reduction scheme one can avoid holes $\left(\mathrm{V}_{\text {red }}^{(16 \rightarrow 8)}\right.$, red dashed-dot curve) or push away nonphysical features due to the PES expansion $\left(\mathrm{V}_{\text {red }}^{(10 \rightarrow 6)}\right.$, blue dashed-dot curve). Results given in Table IV indicate that deviation between vibrational levels for these two latter versions is marginal in the considered energy range. This table clearly shows that the accuracy increases with orders and that the reduction scheme drastically improves the accuracy/cost ratio confirming the results previously obtained for methane $\mathrm{e}^{28,45}$.

After having tested Hamiltonian reductions using quite moderate vibration basis set $F(9)$ in Table IV, we extended our final calculations to larger basis sets $F_{\kappa 1}(13)$ and $F_{\kappa 2}(13)$. We optimized the size of these basis sets by applying supplementary constraints $v_{i} \leq v_{i_{\max }}$ on maximum quantum numbers for individual normal modes.
These limitations as well as $\kappa_{i}$ weight coefficients are summarized in Table V. Convergence tests for basis sets are given in Figure 4 and Table VI that show rms and maximum deviations for vibration levels with respect to the largest one $F_{\kappa 2}(13)$. In this Table we also quote the dimensions of the symmetry blocks before and after the basis set compression. To compute vibrational levels with the largest feasible basis we choose the $H_{10 \rightarrow 6}$ Hamiltonian model which presents an optimal accuracy/cost ratio. With this reduction scheme all the matrix elements with $\sum \Delta v_{i}>6$ have zero values. In case of the $F(9)$ basis only $9 \%$ of $H_{10 \rightarrow 6}$ Hamiltonian matrix elements are non-zero. In frame of our approach the advantage of the primitive harmonic basis set is that the matrices of vibrational Hamiltonian are sparse and the selection rules for both Hamiltonian and dipole moment terms are very simple. But as explain above, for the final vibration-rotation calculation our basis sets are anharmonic ones including inter-mode coupling and resonance mixing. The $F_{\kappa}(N)$ pruning scheme allows quite flexible convergence/cost optimization accounting for each vibrational mode individually. It has been shown previously ${ }^{50}$ that this scheme could provide better accuracy for low vibrations than the "polyad scheme" with the same number of basis functions. In a sense the $F_{\kappa}(N)$ scheme has some similar features to the Multimode one though in Ref. 34 this applies to VSCF basis functions.

$\mathrm{CPU}$ time for the complete calculation of rovibrational spectrum depends of course on the basis set size and on the maximum quantum number $J$. For the $F_{\kappa 2}(13)$ basis, $J=0$ calculation takes about 50 hours per block (8 blocks), including the construction of the reduced $F_{\kappa 2}(13 \rightarrow r)$ basis, on an entire node of "ADA" IBM cluster on IDRIS computer center (32 Intel Sandy Bridge processors and $100 \mathrm{gB}$ of memory). This rather large calculation was necessary to achieve a good convergence for vibrational levels. The $F_{\kappa 2}(13 \rightarrow r)$ basis sets were then used for $J>0$ rovibrational calculation. For the $F_{\kappa 2}(13 \rightarrow 5)$ basis with the maximum $J=25$ quantum number this takes about 65 hours (maximum $A_{g}$ block size of 40000 ) whereas it takes almost 60 hours to compute levels up to $J=10$ with $F_{\kappa 2}(13 \rightarrow 6)$ basis (maximum $A_{g}$ block size of 50 000). Eigenvectors and eigenvalues are calculated with standard routines BLAS/LAPACK for matrix algebra.

\section{Results}

Results for variational calculations of the vibrational levels are presented in Table VII and compared to previous works of Avila and Carrington and Carter et al. ${ }^{34}$ who used a $\operatorname{CCSD}(\mathrm{T}) / \mathrm{cc}-\mathrm{pVTZ}$ PES, and to available experimental data from Ref. 51. As shown in Table VI, the reduction of $F_{\kappa 2}(13)$ basis to $F_{\kappa 2}(13 \rightarrow 6)$ and $F_{\kappa 2}(13 \rightarrow 5)$ permits to reduce the size of hamiltonian matrix blocks by a factor of 40 and 115 respectively. For these bases, a good convergence is achieved for funda- 

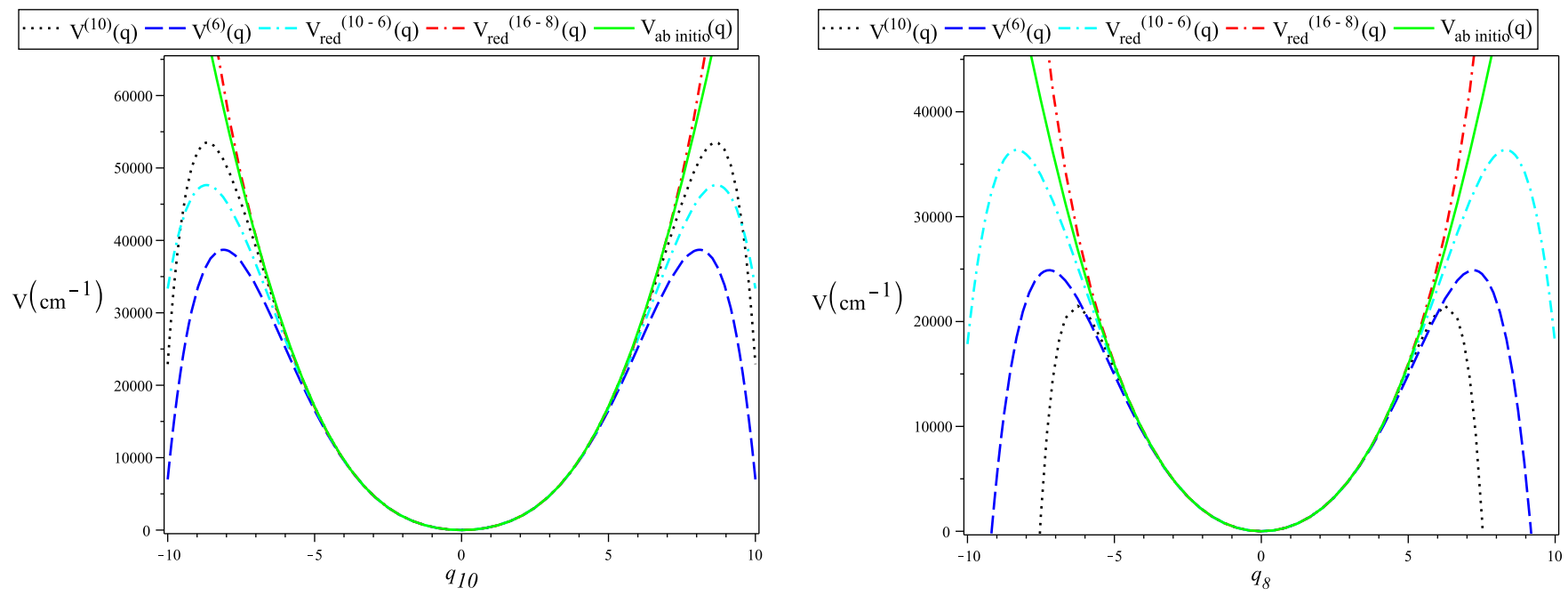

Figure 3. One dimensional cut of the potential expansions $\mathrm{V}_{\text {red }}^{(16 \rightarrow 8)}(\mathrm{q}), \mathrm{V}_{\text {red }}^{(10 \rightarrow 6)}(\mathrm{q}), \mathrm{V}^{(10)}(\mathrm{q}), \mathrm{V}^{(6)}(\mathrm{q})$ and $\mathrm{V}_{a b \text { initio }}(\mathrm{q})$ as a function of bending $\mathrm{q}_{10}$ (left figure) and torsion coordinates $\mathrm{q}_{8}$ (right figure). $\mathrm{V}_{a b}$ initio $(\mathrm{q})$ is obtained without Taylor expansion. $\mathrm{V}_{\text {red }}^{(16 \rightarrow 8)}(\mathrm{q})$ corresponds to $H_{10 \rightarrow 6}^{a} \underset{16 \rightarrow 8}{ }$ of Table IV. It is seen that an optimal choice of the reduction procedure improves the truncated Taylor expansion pushing away non-physical PES features (see text).

Table IV. Dimensions and convergence properties of the Hamiltonian model with respect to $H_{10}$ for ${ }^{12} \mathrm{C}_{2} \mathrm{H}_{4}$.

\begin{tabular}{lccccccc}
\hline & $H_{6}$ & $H_{8 \rightarrow 6}$ & $H_{8}$ & $H_{10 \rightarrow 6}$ & $H_{10 \rightarrow 6}^{a 16 \rightarrow 8}$ & $H_{10 \rightarrow 8}$ & $H_{10}$ \\
\hline $\operatorname{dim}^{a}$ & 9537 & 10865 & 48556 & 10877 & 11300 & 65646 & 184246 \\
time factor $^{b}$ & 1 & 1.1 & 5 & 1.1 & 1.3 & 7 & 19 \\
rms $^{c}$ & 2.21 & 0.48 & 0.41 & 0.29 & 0.28 & 0.025 & - \\
rms $^{d}$ & 2.62 & 0.98 & 0.85 & 0.82 & 0.66 & 0.065 & - \\
\hline
\end{tabular}

See Section IV A for the description of Hamiltonian reduction.

$a$ Dimension of the Hamiltonian model (number of terms in $\{q, p, J\}$ representation).

${ }^{b}$ CPU time factor with respect to computation with $H_{6}$ Hamiltonian model and $F(9)$ basis set.

$c, d \mathrm{rms}$ deviation (in $\mathrm{cm}^{-1}$ ) up to $3200 \mathrm{~cm}^{-1}\left({ }^{c}\right)$ and $6400 \mathrm{~cm}^{-1}\left({ }^{d}\right)$ with respect to variational calculations using

$H_{10}$ Hamiltonian and $F(9)$ basis set.

Table V. Maximum vibrational quantum numbers and weight coefficients for $F_{\kappa 1}(13)$ and $F_{\kappa 2}(13)$ basis sets.

\begin{tabular}{|c|c|c|c|c|c|c|c|c|c|c|c|c|c|}
\hline Basis & Mode i & 1 & 2 & 3 & 4 & 5 & 6 & 7 & 8 & 9 & 10 & 11 & 12 \\
\hline \multirow[t]{2}{*}{$F_{\kappa 1}(13)$} & $\left(v_{i}\right)_{\max }$ & 9 & 10 & 10 & 13 & 9 & 10 & 11 & 11 & 9 & 11 & 9 & 10 \\
\hline & $\kappa_{i}$ & 1.45 & 1.30 & 1.30 & 1.00 & 1.45 & 1.30 & 1.19 & 1.19 & 1.45 & 1.19 & 1.45 & 1.30 \\
\hline \multirow[t]{2}{*}{$F_{\kappa 2}(13)$} & $\left(v_{i}\right)_{\max }$ & 9 & 10 & 11 & 13 & 9 & 11 & 13 & 13 & 9 & 13 & 9 & 10 \\
\hline & $\kappa_{i}$ & 1.45 & 1.30 & 1.19 & 1.00 & 1.45 & 1.19 & 1.00 & 1.00 & 1.45 & 1.00 & 1.45 & 1.30 \\
\hline
\end{tabular}

mentals and first overtones. The two basis sets $F_{\kappa 1}(13)$ and $F_{\kappa 2}(13)$ specified in Table $\mathrm{V}$ differ by the maximum vibrational quantum number for the two lowest bending $\nu_{3}$ and $\nu_{6}$ modes, augmented to $v_{\max }=11$, and all torsion modes adjusted to $v_{\max }=13$. As the sizes of matrices become larger as $J$ increases, we have adopted a strategy which consists in partitioning the rovibrational problem as follows: for $J=0$ calculations were performed with $F_{\kappa 2}(13)$, for $1 \leq J \leq 10$ with $F_{\kappa 2}(13 \rightarrow 6)$ and for $11 \leq J \leq 20$ with $F_{\kappa 2}(13 \rightarrow 5)$. For the two latter basis sets, a vibrational VSS cut-off $E_{\max }$ has been fixed to $16000 \mathrm{~cm}^{-1}$ with respect to zero-point vibrational energy. Obviously with such a cut-off we consider almost all vibrational wavefunctions of $F_{\kappa 1}(13)$ and $F_{\kappa 2}(13)$, i.e. there is no compression of the basis with respect to this cut-off.

A comparison of vibrational band centers with those predicted from the previous potential energy surface of Martin et $a l .{ }^{33}$, obtained at the CCSD(T)/cc-pVTZ PES level of $a b$ initio calculations, shows a significant improvement for both fundamentals and overtones, and generally of all bands in the considered region. This could be possibly explained by increasing cardinal number of the electronic basis set but also by the larger grid of nuclear configurations included in our study. This permits accounting for higher-order terms in the analytical 
Table VI. Dimensions and convergence properties of the $F\left(v_{\max }\right)$ basis with respect to the largest $F_{\kappa 2}(13)$ set using the $H_{10 \rightarrow 6}$ Hamiltonian model ${ }^{a}$ for ${ }^{12} \mathrm{C}_{2} \mathrm{H}_{4}$.

\begin{tabular}{lcccccccc}
\hline Basis & $F(8)$ & $F(9)$ & $F_{\kappa 1}(13 \rightarrow 5)$ & $F_{\kappa 2}(13 \rightarrow 5)$ & $F_{\kappa 1}(13 \rightarrow 6)$ & $F_{\kappa 2}(13 \rightarrow 6)$ & $F_{\kappa 1}(13)$ & $F_{\kappa 2}(13)$ \\
\hline$N_{A_{g}}$ & 17000 & 38970 & 922 & 922 & 2654 & 2654 & 66039 & 106889 \\
$N_{A_{u}}$ & 14890 & 35120 & 690 & 690 & 2122 & 2122 & 61814 & 101265 \\
$N_{B_{1 g}}$ & 14650 & 34670 & 646 & 646 & 2050 & 2050 & 61087 & 100366 \\
$N_{B_{1 u}}$ & 16550 & 38310 & 850 & 850 & 2498 & 2498 & 65008 & 105518 \\
$N_{B_{2 g}}$ & 16550 & 38310 & 850 & 850 & 2498 & 2498 & 65084 & 105643 \\
$N_{B_{2 u}}$ & 14890 & 35120 & 690 & 690 & 2122 & 2122 & 61697 & 101145 \\
$N_{B_{3 g}}$ & 14890 & 35120 & 690 & 690 & 2122 & 2122 & 61729 & 101255 \\
$N_{B_{3 u}}$ & 16550 & 38310 & 850 & 850 & 2498 & 2498 & 65121 & 105697 \\
rms $^{b}$ & 1.51 & 0.54 & 0.20 & - & 0.20 & - & 0.20 & - \\
rms $^{c}$ & 6.66 & 1.96 & 0.71 & - & 0.71 & - & 0.71 & - \\
max $^{d}$ & 19.07 & 6.83 & 1.52 & - & 1.52 & - & 1.52 & - \\
\hline
\end{tabular}

$a$ Notation $10 \rightarrow 6$ means that the Hamiltonian is expanded up to $10^{\text {th }}$ power in $q$ and converted to $6^{\text {th }}$ power as described in Section IV A; $N_{\Gamma}$ denote dimensions of symmetry blocks for $N_{\Gamma}=$ $A_{g}, A_{u}, B_{1 g}, B_{1 u}, B_{2 g}, B_{2 u}, B_{3 g}$ and $B_{3 u}$.

$b, c, d$ maximum $\left(^{d}\right)$ and rms deviation (in $\mathrm{cm}^{-1}$ ) up to $3200 \mathrm{~cm}^{-1}\left({ }^{b}\right)$ and $6400 \mathrm{~cm}^{-1}\left({ }^{c}\right)$ with respect to variational calculations with $F_{\kappa 2}(13)$ basis.

By construction, in the VSS procedure the vibrational eigenvalues associated to $F_{\kappa}(N \rightarrow r)$ and $F_{\kappa}(N)$ basis sets are taken equal. Consequently for $J=0$ the rms deviation between the corresponding eigenvalues sets is zero.

PES representation. Comparing now the results obtained with our new PES and experimental data, we can see that these purely $a b$ initio results are in a quite good agreement with observed bands, with the rms deviation

of less than $2.7 \mathrm{~cm}^{-1}$ for fundamentals and $4 \mathrm{~cm}^{-1}$ for first overtones.

Table VII: Comparison of ab initio calculations of ${ }^{12} \mathrm{C}_{2} \mathrm{H}_{4}$ vibrational energy levels (this work $=\mathrm{TW}$ ) using $F_{\kappa 2}(13)$ basis with Avila and Carrington ${ }^{32}$, Carter et $a .^{34}$, and available experimental values ${ }^{51}$ up to $7800 \mathrm{~cm}^{-1}$.

\begin{tabular}{|c|c|c|c|c|c|c|c|c|c|}
\hline Band & Ref. 32 & Ref. 34 & $\overline{\mathrm{TW}^{a}}$ & Exp. $^{b}$ & Band & Ref. 32 & Ref. 34 & $\overline{\mathrm{TW}^{a}}$ & Exp. $^{b}$ \\
\hline$z p e$ & 11004.79 & 11003.98 & 11014.91 & & & & & & \\
\hline$\nu_{10}$ & 821.74 & 821.15 & 822.42 & 825.92 & $\nu_{8}$ & 926.81 & 926.13 & 934.29 & 939.86 \\
\hline$\nu_{7}$ & 947.22 & 946.49 & 949.51 & 948.77 & $\nu_{4}$ & 1025.93 & 1025.28 & 1024.94 & 1025.58 \\
\hline$\nu_{12}$ & 1440.20 & 1439.67 & 1441.11 & 1442.44 & $\nu_{2}$ & 1623.53 & 1623.17 & 1624.43 & 1626.17 \\
\hline $2 \nu_{10}$ & 1655.51 & 1654.22 & 1658.39 & 1664.16 & $\nu_{8}+\nu_{10}$ & 1751.09 & 1750.05 & 1757.70 & 1765.78 \\
\hline$\nu_{7}+\nu_{10}$ & 1775.36 & 1774.18 & 1778.34 & 1781.01 & $\nu_{4}+\nu_{10}$ & 1849.44 & 1848.32 & 1848.61 & 1851.51 \\
\hline$\nu_{4}+\nu_{7}$ & 1964.46 & 1961.19 & 1966.50 & 1965.44 & $\nu_{6}+\nu_{10}$ & 2040.33 & 2038.34 & 2041.34 & 2047.76 \\
\hline $2 \nu_{4}$ & 2049.11 & 2047.54 & 2046.44 & 2046.40 & $\nu_{6}+\nu_{8}$ & 2158.35 & 2157.66 & 2163.12 & \\
\hline$\nu_{3}+\nu_{10}$ & 2165.84 & 2164.88 & 2167.19 & 2172.50 & $\nu_{6}+\nu_{7}$ & 2173.18 & 2172.44 & 2175.40 & \\
\hline$\nu_{4}+\nu_{6}$ & 2250.59 & 2249.87 & 2249.08 & 2251.50 & $\nu_{10}+\nu_{12}$ & 2259.26 & 2256.73 & 2260.75 & \\
\hline$\nu_{3}+\nu_{8}$ & 2264.23 & 2263.77 & 2273.84 & & $\nu_{3}+\nu_{7}$ & 2285.87 & 2285.37 & 2289.89 & 2291.50 \\
\hline$\nu_{8}+\nu_{12}$ & 2362.40 & 2361.04 & 2363.49 & & $\nu_{3}+\nu_{4}$ & 2363.47 & 2363.20 & 2372.52 & \\
\hline$\nu_{3}+\nu_{6}$ & 2562.94 & 2562.51 & 2564.39 & & $\nu_{2}+\nu_{7}$ & 2564.38 & 2564.04 & 2569.48 & 2571.00 \\
\hline$\nu_{8}+2 \nu_{10}$ & 2585.30 & 2583.95 & 2593.75 & & $\nu_{7}+2 \nu_{10}$ & 2612.42 & 2610.83 & 2618.33 & \\
\hline$\nu_{2}+\nu_{4}$ & 2646.03 & 2645.42 & 2646.09 & & $\nu_{6}+\nu_{12}$ & 2659.38 & 2658.13 & 2659.40 & \\
\hline $2 \nu_{3}$ & 2680.89 & 2680.54 & 2683.05 & 2685.30 & $\nu_{4}+2 \nu_{10}$ & 2683.77 & 2682.43 & 2685.01 & \\
\hline $2 \nu_{8}+\nu_{10}$ & 2683.88 & 2682.68 & 2698.44 & & $\nu_{7}+\nu_{8}+\nu_{10}$ & 2699.66 & 2699.05 & 2714.55 & \\
\hline $2 \nu_{7}+\nu_{10}$ & 2729.95 & 2728.67 & 2737.25 & & $\nu_{3}+\nu_{12}$ & 2774.95 & 2774.10 & 2778.00 & \\
\hline$\nu_{4}+\nu_{8}+\nu_{10}$ & 2776.23 & 2775.76 & 2778.04 & & $3 \nu_{8}$ & 2784.91 & 2782.78 & 2796.68 & \\
\hline$\nu_{7}+2 \nu_{8}$ & 2791.98 & 2786.59 & 2818.31 & 2829.80 & $\nu_{4}+\nu_{7}+\nu_{10}$ & 2794.08 & 2793.63 & 2826.52 & \\
\hline $2 \nu_{7}+\nu_{8}$ & 2812.72 & 2808.06 & 2833.87 & & $\nu_{2}+\nu_{6}$ & 2832.77 & 2831.56 & 2840.09 & \\
\hline $3 \nu_{7}$ & 2843.78 & 2842.37 & 2856.80 & 2854.00 & $\nu_{6}+2 \nu_{10}$ & 2867.62 & 2864.92 & 2871.07 & \\
\hline
\end{tabular}


Table VII: (Continued)

\begin{tabular}{|c|c|c|c|c|c|c|c|c|c|}
\hline$\overline{\text { Band }}$ & Ref. 32 & Ref. 34 & $\overline{\mathrm{TW}^{a}}$ & Exp. $^{b}$ & Band & Ref. 32 & Ref. 34 & $\mathrm{TW}^{a}$ & Exp. $^{b}$ \\
\hline $2 \nu_{12}$ & 2872.07 & 2870.54 & 2871.68 & 2877.30 & $2 \nu_{4}+\nu_{10}$ & 2874.62 & 2873.11 & 2874.66 & \\
\hline$\nu_{4}+2 \nu_{8}$ & 2875.25 & 2868.85 & 2887.43 & & $\nu_{4}+\nu_{7}+\nu_{8}$ & 2883.78 & 2874.24 & 2898.17 & \\
\hline$\nu_{4}+2 \nu_{7}$ & 2903.83 & 2899.08 & 2911.66 & & $\nu_{2}+\nu_{3}$ & 2957.97 & 2957.84 & 2959.07 & 2961.60 \\
\hline $2 \nu_{4}+\nu_{7}$ & 2979.46 & 2973.80 & 2982.16 & & $\nu_{11}$ & 2983.94 & 2985.64 & 2985.38 & 2988.63 \\
\hline$\nu_{6}+\nu_{7}+\nu_{10}$ & 2996.74 & 2997.95 & 2999.83 & & $\nu_{3}+2 \nu_{10}$ & 3000.26 & 2998.82 & 3002.86 & \\
\hline$\nu_{1}$ & 3019.66 & 3020.15 & 3018.99 & 3021.85 & $\nu_{4}+\nu_{6}+\nu_{10}$ & 3068.90 & 3069.92 & 3064.61 & \\
\hline $2 \nu_{10}+\nu_{12}$ & 3094.98 & 3092.90 & 3100.04 & 3104.33 & $\nu_{6}+2 \nu_{8}$ & 3095.86 & 3095.34 & 3101.69 & \\
\hline$\nu_{9}$ & 3098.04 & 3098.70 & 3101.69 & 3104.87 & $\nu_{6}+\nu_{7}+\nu_{8}$ & 3105.14 & 3107.71 & 3115.43 & \\
\hline$\nu_{3}+\nu_{7}+\nu_{10}$ & 3117.04 & 3119.23 & 3121.54 & & $\nu_{6}+2 \nu_{7}$ & 3123.62 & 3122.88 & 3129.32 & \\
\hline$\nu_{4}+\nu_{6}+\nu_{8}$ & 3182.06 & 3181.95 & 3181.95 & & $\nu_{8}+\nu_{10}+\nu_{12}$ & 3184.69 & 3185.11 & 3189.86 & \\
\hline$\nu_{3}+2 \nu_{8}$ & 3189.21 & 3188.39 & 3192.49 & & $\nu_{3}+\nu_{4}+\nu_{10}$ & 3189.56 & 3192.03 & 3193.74 & \\
\hline$\nu_{4}+\nu_{6}+\nu_{7}$ & 3191.15 & 3191.34 & 3210.44 & & $\nu_{3}+\nu_{7}+\nu_{8}$ & 3203.64 & 3204.24 & 3214.14 & \\
\hline$\nu_{3}+\nu_{4}+\nu_{8}$ & 3283.44 & 3284.04 & 3288.87 & & $2 \nu_{8}+\nu_{12}$ & 3285.89 & 3284.30 & 3289.05 & \\
\hline$\nu_{4}+\nu_{10}+\nu_{12}$ & 3289.40 & 3289.81 & 3303.16 & & $\nu_{7}+\nu_{8}+\nu_{12}$ & 3297.05 & 3296.89 & 3309.51 & 3327.30 \\
\hline$\nu_{3}+\nu_{4}+\nu_{7}$ & 3299.32 & 3300.35 & 3320.87 & & $2 \nu_{7}+\nu_{12}$ & 3322.38 & 3320.95 & 3336.46 & \\
\hline $4 \nu_{10}$ & 3340.58 & 3341.94 & 3351.50 & & $\nu_{2}+\nu_{8}+\nu_{10}$ & 3357.40 & 3359.81 & 3367.05 & \\
\hline$\nu_{3}+\nu_{6}+\nu_{10}$ & 3382.29 & 3381.94 & 3381.07 & & $\nu_{3}+2 \nu_{4}$ & 3382.80 & 3382.51 & 3384.25 & \\
\hline$\nu_{2}+\nu_{7}+\nu_{10}$ & 3383.76 & 3386.10 & 3388.90 & & $\nu_{4}+\nu_{8}+\nu_{12}$ & 3387.16 & 3385.90 & 3390.74 & \\
\hline $2 \nu_{6}+\nu_{8}$ & 3390.24 & 3389.20 & 3393.14 & & $2 \nu_{6}+\nu_{7}$ & 3399.30 & 3398.20 & 3400.15 & \\
\hline$\nu_{4}+\nu_{7}+\nu_{12}$ & 3399.91 & 3398.95 & 3405.46 & & $\nu_{8}+3 \nu_{10}$ & 3426.08 & 3426.54 & 3437.52 & \\
\hline$\nu_{7}+3 \nu_{10}$ & 3456.40 & 3456.41 & 3459.91 & & $\nu_{2}+\nu_{4}+\nu_{10}$ & 3459.82 & 3461.79 & 3466.76 & \\
\hline$\nu_{2}+2 \nu_{8}$ & 3462.02 & 3462.25 & 3472.04 & 3496.90 & $\nu_{6}+\nu_{10}+\nu_{12}$ & 3473.49 & 3470.48 & 3474.54 & 3480.10 \\
\hline$\nu_{4}+2 \nu_{6}$ & 3475.38 & 3474.25 & 3488.05 & & $\nu_{2}+\nu_{7}+\nu_{8}$ & 3476.20 & 3478.08 & 3490.41 & 3504.59 \\
\hline $2 \nu_{4}+\nu_{12}$ & 3493.21 & 3491.55 & 3500.06 & & $\nu_{3}+\nu_{6}+\nu_{8}$ & 3493.69 & 3496.31 & 3500.53 & \\
\hline$\nu_{7}+2 \nu_{10}+\nu_{12}$ & 4043.17 & 4043.91 & 4054.67 & 4057.72 & $4 \nu_{4}$ & 4087.53 & 4086.85 & 4079.89 & 4076.00 \\
\hline$\nu_{4}+\nu_{9}$ & - & - & 4119.87 & 4129.40 & $\nu_{6}+\nu_{11}$ & - & - & 4199.86 & 4206.59 \\
\hline$\nu_{2}+\nu_{6}+\nu_{12}$ & - & - & 4284.02 & 4288.41 & $\nu_{2}+2 \nu_{8}+\nu_{10}$ & - & - & 4303.53 & 4297.50 \\
\hline$\nu_{5}+\nu_{6}$ & - & - & 4302.81 & 4310.10 & $\nu_{3}+2 \nu_{4}+\nu_{7}$ & - & - & 4314.32 & 4311.40 \\
\hline$\nu_{6}+2 \nu_{1} 0+\nu_{12}$ & - & - & 4308.11 & 4312.67 & $\nu_{6}+\nu_{9}$ & - & - & 4316.23 & 4321.77 \\
\hline$\nu_{3}+\nu_{11}$ & - & - & 4323.42 & 4328.65 & $\nu_{3}+\nu_{6}+\nu_{8}+\nu_{10}$ & - & - & 4322.80 & 4332.40 \\
\hline$\nu_{11}+\nu_{12}$ & - & - & 4401.84 & 4407.70 & $\nu_{2}+\nu_{3}+\nu_{12}$ & - & - & 4409.99 & 4411.00 \\
\hline$\nu_{3}+\nu_{9}$ & - & - & 4435.67 & 4439.79 & $\nu_{1}+\nu_{12}$ & - & - & 4455.94 & 4460.03 \\
\hline$\nu_{4}+\nu_{6}+\nu_{10}+\nu_{12}$ & - & - & 4503.39 & 4508.90 & $\nu_{5}+\nu_{12}$ & - & - & 4509.94 & 4514.65 \\
\hline $2 \nu_{3}+\nu_{7}+\nu_{8}$ & - & - & 4559.26 & 4567.60 & $\nu_{2}+\nu_{11}$ & - & - & 4592.11 & 4596.89 \\
\hline$\nu_{1}+\nu_{2}$ & - & - & 4628.01 & 4631.80 & $\nu_{2}+2 \nu_{10}+\nu_{12}$ & - & - & 4698.07 & 4700.00 \\
\hline$\nu_{2}+\nu_{9}$ & - & - & 4724.45 & 4729.75 & $\nu_{7}+\nu_{10}+\nu_{11}$ & - & - & 4751.01 & 4748.70 \\
\hline$\nu_{9}+2 \nu_{10}$ & - & - & 4754.97 & 4764.24 & $\nu_{1}+2 \nu_{8}$ & - & - & 4874.67 & 4887.10 \\
\hline $2 \nu_{4}+\nu_{11}$ & - & - & 5022.36 & 5025.00 & $\nu_{1}+\nu_{6}+\nu_{10}$ & - & - & 5044.42 & 5058.50 \\
\hline$\nu_{5}+\nu_{6}+\nu_{10}$ & - & - & 5115.20 & 5124.00 & $\nu_{5}+\nu_{6}+\nu_{7}$ & - & - & 5236.39 & 5245.00 \\
\hline $2 \nu_{6}+\nu_{11}$ & - & - & 5411.16 & 5425.00 & $\nu_{4}+\nu_{9}+\nu_{12}$ & - & - & 5554.21 & 5556.00 \\
\hline$\nu_{1}+\nu_{6}+\nu_{12}$ & - & - & 5667.51 & 5672.00 & $\nu_{5}+\nu_{6}+\nu_{12}$ & - & - & 5727.70 & 5735.00 \\
\hline$\nu_{1}+\nu_{3}+\nu_{12}$ & - & - & 5782.24 & 5788.00 & $3 \nu_{8}+\nu_{11}$ & - & - & 5789.08 & 5792.00 \\
\hline$\nu_{2}+\nu_{6}+\nu_{11}$ & - & - & 5791.86 & 5800.00 & $\nu_{2}+\nu_{6}+\nu_{9}$ & - & - & 5923.64 & 5918.77 \\
\hline$\nu_{2}+\nu_{3}+\nu_{11}$ & - & - & 5914.00 & 5927.57 & $2 \nu_{11}$ & - & - & 5934.83 & 5939.40 \\
\hline$\nu_{5}+\nu_{6}+2 \nu_{10}$ & - & - & 5947.13 & 5953.70 & $\nu_{1}+\nu_{2}+\nu_{3}$ & - & - & 5952.03 & 5960.30 \\
\hline$\nu_{5}+\nu_{11}$ & - & - & 5989.60 & 5994.79 & $\nu_{1}+\nu_{2}+\nu_{12}$ & - & - & 6065.39 & 6071.58 \\
\hline
\end{tabular}


Table VII: (Continued)

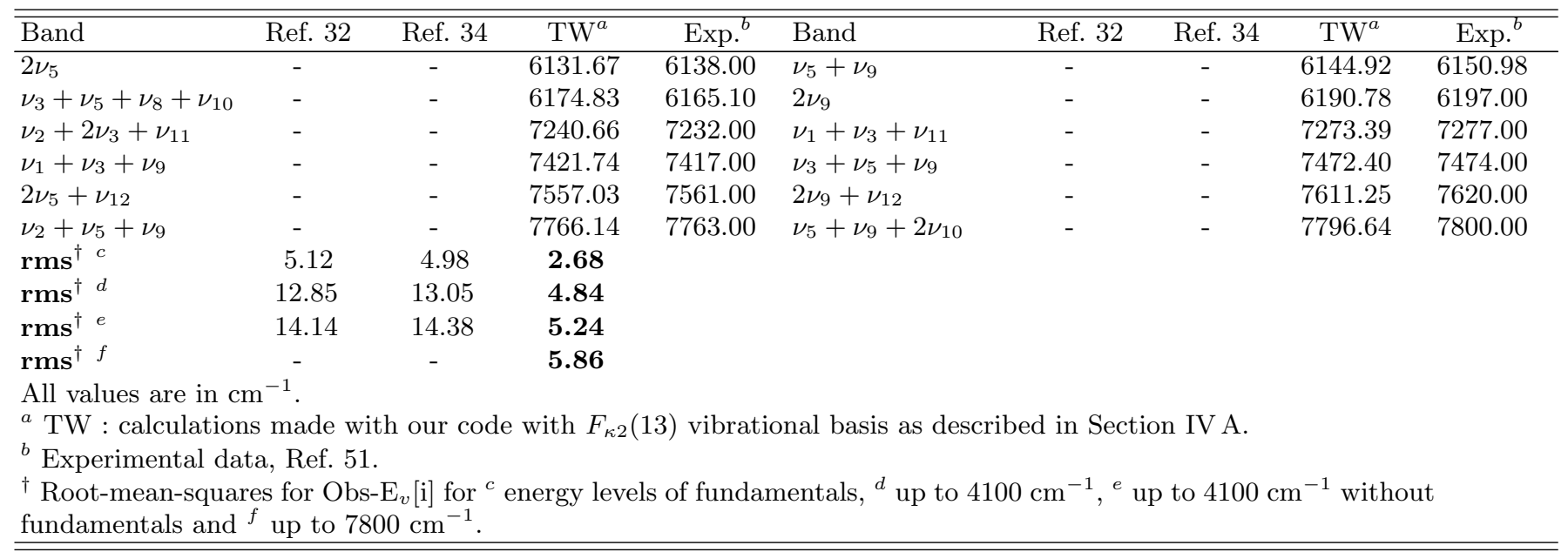

\section{CALCULATION OF ${ }^{12} \mathbf{C}_{2} \mathbf{D}_{4}$ AND ${ }^{13} \mathbf{C}_{2} \mathbf{H}_{4}$ VIBRATION LEVELS}

An extension of the vibrational energy levels calculation for ${ }^{12} \mathrm{C}_{2} \mathrm{H}_{4}$ was made for some isotopologues to test the accuracy of the PES. Isotopic species ${ }^{12} \mathrm{C}_{2} \mathrm{D}_{4}$ and ${ }^{13} \mathrm{C}_{2} \mathrm{H}_{4}$ are considered because (i) the $D_{2 h}$ symmetry is not changed and (ii) there exists some experimental data for these species. For the calculation of vibration levels of these two species, a quite similar technique was applied. We used the same sixth order expansion of the ab initio PES as for ${ }^{12} \mathrm{C}_{2} \mathrm{H}_{4}$ expressed in internal coordinates and we substituted the atomic masses in the kinetic energy operator when running the above described program for energy levels calculation. The same equilibrium geometry as for ${ }^{12} \mathrm{C}_{2} \mathrm{H}_{4}$ was also kept. For this work, the eigenvectors $F(9)$ basis set was used to compute vibrational line positions that is sufficient to well converge $J=0$ fundamentals of both isotopic species, at least up to energies necessary for the comparison with the available observations.

In Tables VIII and IX we give calculated vibration levels as well as the comparison with the corresponding observed band centres, when available. For both species very few bands are known: concerning the ${ }^{12} \mathrm{C}_{2} \mathrm{D}_{4}$ isotopologue, Duncan et al. ${ }^{60}$ and later Tan et al. ${ }^{54,55,61,62}$ have analyzed most fundamentals and some combination bands, however for ${ }^{13} \mathrm{C}_{2} \mathrm{H}_{4}$ the situation is much worse and only a few fundamentals are known ${ }^{58,59}$. In general the agreement with observations is very good for both isotopic species that confirms the validity of our $a b$ initio PES. The rms deviation between theoretically calculated and observed band centres is $4.8 \mathrm{~cm}^{-1}\left(2.4 \mathrm{~cm}^{-1}\right.$ for fundamentals) for ${ }^{12} \mathrm{C}_{2} \mathrm{D}_{4}$ and $<3 \mathrm{~cm}^{-1}$ for ${ }^{13} \mathrm{C}_{2} \mathrm{H}_{4}$. Calculated isotopic shifts are also given in Table VIII and are in very good agreement with isotopic shifts derived from observed data.

\section{ROTATIONAL LEVELS OF ${ }^{12} \mathbf{C}_{2} \mathbf{H}_{4}$}

Theoretical predictions of rotational and rovibrational energy levels of penta- and hexa-atomic molecules by means of variational calculations with the precision required for high-resolution spectroscopy remains a formidable challenge. In this section we consider a validation of our new PES with respect to pure rotational and rovibrational calculations for the $\nu_{9} / \nu_{11}$ bands up to $J=20$. It is well-known that ground state rotational energies are essentially determined by the equilibrium geometry and by the shape of the PES at the bottom of the potential well. Our previous experience in computation of rotational levels of other systems such as $\mathrm{CH}_{4}$ or $\mathrm{PH}_{3}$ using various $a b$ initio $\mathrm{PESs}^{18,26,63}$ suggested that rotational levels $J=1$ are less sensitive to small variations of the PESs force constants but could strongly depend on relatively small errors in the equilibrium geometry. As shown in Table I, test calculations achieved at various levels of $a b$ initio theory pointed out that pure $a b$ initio equilibrium geometries did not give a sufficient accuracy for rotational levels of $\mathrm{C}_{2} \mathrm{H}_{4}$. Therefore we proceeded in this work by an empirical optimization of the equilibrium nuclear configuration for our $\operatorname{CCSD}(\mathrm{T}) / \mathrm{cc}-$ pVQZ PES in order to improve rotational predictions, as described in Section II A, in a similar strategy as that used by Pawlowski et $a .^{40}$ to obtain reliable equilibrium structures. This optimization leads to a set of three parameters of equilibrium geometry used for the potential expansion in the Hamiltonian. For the calculation of rovibrational energies, the matrix elements of this Hamiltonian operator are obtained in the coupled basis written as a product between the vibrational functions defined in Section IV A and rotational functions as

$$
\left|\left(\Psi_{v}^{C_{v}} \times \Psi_{r}^{J, n C_{r}}\right)^{(C)}\right\rangle,
$$



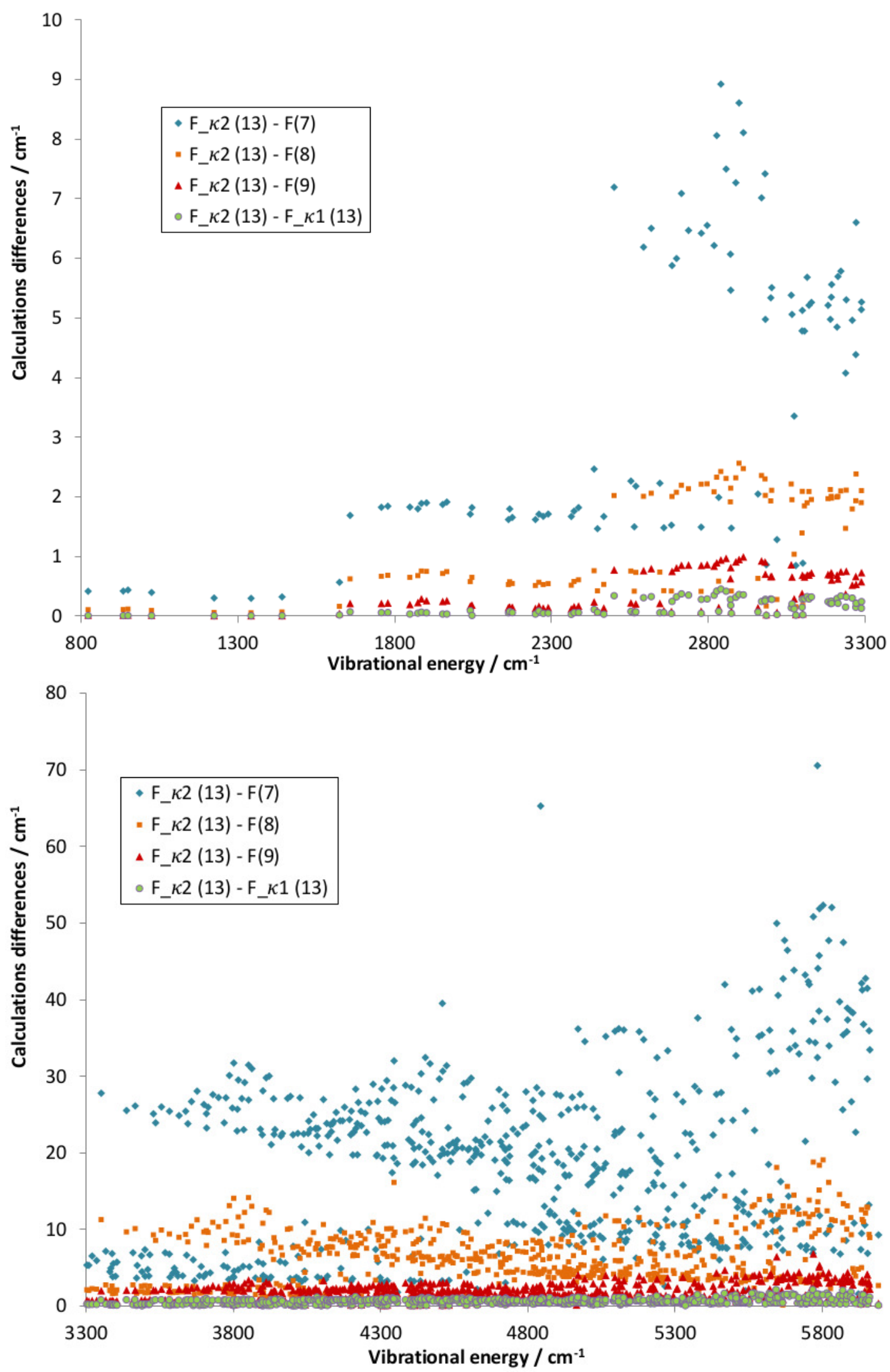

Figure 4. Convergence of ethylene vibration levels using different vibrational basis set. Discrepancies between calculations with $F(7), F(8), F(9)$ and $F_{\kappa 1}(13)$, and the reference $F_{\kappa 2}(13)$ basis are given versus band centers.

where $\Psi_{r}^{J, n C_{r}}$ is the rotational basis related to standard basis through
$C_{v}, C_{r}$ and $C$ are the vibrational, rotational and rovibrational symmetry species in $D_{2 h}$ group, respectively.

$$
\left|\Psi_{r}^{J, n C_{r}}\right\rangle=\sum^{(J)} G_{n C}^{K}|J, K\rangle .
$$


Table VIII. Comparison of band centres of ${ }^{12} \mathrm{C}_{2} \mathrm{D}_{4}$ molecule calculated in this work (Calc., F(9) basis) with observations (Obs.). Calculated and experimentally derived isotopic shifts are reported.

\begin{tabular}{|c|c|c|c|c|c|c|c|c|}
\hline \multirow[b]{2}{*}{ Band } & \multicolumn{3}{|c|}{${ }^{12} \mathrm{C}_{2} \mathrm{D}_{4}$} & \multicolumn{2}{|c|}{${ }^{12} \mathrm{C}_{2} \mathrm{H}_{4}$} & \multicolumn{3}{|c|}{ Isotopic shifts $\Delta$} \\
\hline & Obs. $^{a}$ & Calc. & Obs-Calc. & Obs. & Calc. & $\Delta$ Calc. $^{b}$ & $\Delta$ Obs. $^{b}$ & $\Delta$ Obs.-Calc. \\
\hline$\nu_{10}$ & $593.34^{52}$ & 590.79 & 2.55 & 825.92 & 822.23 & 231.44 & 232.58 & 1.14 \\
\hline$\nu_{7}$ & $719.77^{52}$ & 720.45 & -0.68 & 948.77 & 949.51 & 229.06 & 229.00 & -0.06 \\
\hline$\nu_{4}$ & $729.96^{52}$ & 729.33 & 0.63 & 1025.58 & 1024.93 & 295.60 & 295.62 & 0.02 \\
\hline$\nu_{8}$ & $780.00^{33}$ & 774.06 & 5.94 & 939.86 & 934.42 & 160.36 & 159.86 & -0.50 \\
\hline$\nu_{3}$ & 984.60 & 984.48 & 0.12 & 1343.31 & 1342.28 & 357.80 & 358.71 & 0.91 \\
\hline$\nu_{6}$ & $1001.10^{53}$ & 1001.51 & -0.41 & 1225.41 & 1224.09 & 222.58 & 224.31 & 1.73 \\
\hline$\nu_{12}$ & $1076.98^{54}$ & 1075.70 & 1.28 & 1442.44 & 1440.68 & 364.99 & 365.46 & 0.47 \\
\hline$\nu_{2}$ & $1518.00^{33}$ & 1516.55 & 1.45 & 1626.17 & 1624.18 & 107.64 & 108.17 & 0.53 \\
\hline$\nu_{11}$ & 2201.00 & 2198.44 & 2.56 & 2988.63 & 2985.35 & 786.91 & 787.63 & 0.72 \\
\hline$\nu_{1}$ & 2261.60 & 2260.21 & 1.39 & 3021.85 & 3019.06 & 758.85 & 760.25 & 1.40 \\
\hline$\nu_{5}$ & 2315.40 & 2312.47 & 2.93 & 3082.36 & 3079.78 & 767.31 & 766.96 & -0.35 \\
\hline$\nu_{9}$ & $2341.84^{55}$ & 2339.24 & 2.60 & 3104.33 & 3101.65 & 762.41 & 762.49 & 0.08 \\
\hline$\nu_{3}+\nu_{12}$ & 2060.00 & 2054.96 & 5.04 & & 2777.46 & 722.50 & & \\
\hline$\nu_{11}+2 \nu_{12}$ & 4342.00 & 4334.58 & 7.41 & & 5813.59 & 1479.01 & & \\
\hline$\nu_{1}+\nu_{3}+\nu_{12}$ & 4316.00 & 4308.38 & 7.62 & 5788.00 & 5781.40 & 1473.01 & 1472.00 & -1.01 \\
\hline$\nu_{1}+\nu_{11}$ & 4428.60 & 4426.55 & 2.05 & & 5945.79 & 1519.24 & & \\
\hline$\nu_{5}+\nu_{11}$ & 4476.20 & 4471.45 & 4.75 & 5994.79 & 5989.45 & 1518.00 & 1518.59 & 0.59 \\
\hline$\nu_{5}+\nu_{9}$ & $4626.82^{56}$ & 4621.54 & 5.28 & 6150.98 & 6144.84 & 1523.31 & 1524.16 & 0.85 \\
\hline $2 \nu_{11}+\nu_{3}+\nu_{12}$ & 6424.00 & 6418.04 & 5.96 & & & & & \\
\hline $3 \nu_{11}$ & 6538.00 & 6535.51 & 2.49 & & & & & \\
\hline $2 \nu_{1}+\nu_{3}+\nu_{12}$ & 6542.00 & 6549.89 & -7.89 & & & & & \\
\hline$\nu_{9}+2 \nu_{11}$ & 6625.30 & 6623.65 & 1.65 & & & & & \\
\hline $2 \nu_{1}+\nu_{11}$ & 6640.00 & 6639.66 & 0.34 & & & & & \\
\hline $2 \nu_{5}+\nu_{3}+\nu_{12}$ & 6649.00 & 6640.97 & 8.03 & & & & & \\
\hline $2 \nu_{9}+\nu_{3}+\nu_{12}$ & 6710.00 & 6699.69 & 10.31 & & & & & \\
\hline $2 \nu_{5}+\nu_{11}$ & 6742.30 & 6738.56 & 3.74 & & & & & \\
\hline$\nu_{5}+\nu_{9}+2 \nu_{12}$ & 6755.50 & 6750.97 & 4.53 & & & & & \\
\hline $2 \nu_{9}+\nu_{11}$ & 6805.10 & 6800.93 & 4.17 & & & & & \\
\hline$\nu_{1}+\nu_{5}+\nu_{9}$ & 6864.50 & 6861.65 & 2.85 & & & & & \\
\hline $2 \nu_{5}+\nu_{9}$ & 6889.00 & 6881.49 & 7.51 & & & & & \\
\hline $3 \nu_{9}$ & 6987.0 & 6979.80 & 7.70 & & & & & \\
\hline $\mathbf{r m s}^{c}$ & & & 2.42 & & & & & \\
\hline $\mathbf{r m s}^{d}$ & & & 4.81 & & & & & \\
\hline
\end{tabular}

All values are in $\mathrm{cm}^{-1}$.

${ }^{a}$ From Ref. 57 if no other reference precised.

${ }^{b}$ Isotopic shifts calculated as $\Delta=E\left({ }^{12} \mathrm{C}_{2} \mathrm{H}_{4}\right)-E\left({ }^{12} \mathrm{C}_{2} \mathrm{D}_{4}\right)$.

${ }^{c}$ Root-mean-squares (Obs.-Calc.) deviation for fundamentals.

${ }^{d}$ Root-mean-squares (Obs.-Calc.) deviation with respect of all observed data.

The $G$ matrix corresponds to the transformation matrix from $S O(3)$ to $D_{2 h}{ }^{64}$ and is nothing but a Wang transformation ${ }^{65}$. With such a formalism, all the rovibrational levels are described by $(J, n C)$ labels, where $n$ is the multiplicity index of the $C$ irreducible representation, associated to $|K|$. The correspondence with the standard $K_{a}, K_{c}$ indices can be easily done ${ }^{65}$.

In this work, rovibrational functions, built as described above and in Section IV A, are reduced functions $F_{\kappa 2}(13 \rightarrow 6)$ for $1 \leq J \leq 10$ and $F_{\kappa 2}(13 \rightarrow 5)$ for $11 \leq J \leq 20$. Vibrational $J=0$ levels were matched to observed band centers ${ }^{8}$ using the VSS empirical corrections. This allows focusing more precisely on the ro- tational dependence of residual errors with respect to $J$ quantum number as plotted in Figure 5 up to $J=5$. Such an empirical optimization represents a pragmatic method for improving $a b$ initio predictions for spectroscopic applications. Figure 5 shows the improvement of rotational levels computed from our PES (using pVQZ and pCVQZ equilibrium geometries) with respect to the previous "morsified" version ${ }^{32}$ of the CCSD $(\mathrm{T}) / \mathrm{cc}-\mathrm{pVTZ}$ PES originally computed by Martin ${ }^{33}$. Figure 6 gives a similar comparison for upper vibration-rotation levels of the $\nu_{9} / \nu_{11}$ bands, the pure vibrational error being substracted for all versions of calculations as described above. 
Table IX. Comparison of band centres of ${ }^{13} \mathrm{C}_{2} \mathrm{H}_{4}$ molecule calculated in this work (Calc., $F(9)$ basis) with observations (Obs.) $)^{58,59}$. Calculated and experimentally derived isotopic shifts are reported.

\begin{tabular}{|c|c|c|c|c|c|c|c|c|}
\hline \multirow[b]{2}{*}{ Band } & \multicolumn{3}{|c|}{${ }^{13} \mathrm{C}_{2} \mathrm{H}_{4}$} & \multicolumn{2}{|c|}{${ }^{12} \mathrm{C}_{2} \mathrm{H}_{4}$} & \multicolumn{3}{|c|}{ Isotopic shifts $\Delta$} \\
\hline & Obs. & Calc. & Obs-Calc. & Obs. & Calc. & $\Delta$ Calc. & $\Delta \mathrm{Obs}$ & $\Delta$ Obs-Calc. \\
\hline$\nu_{10}$ & & 821.22 & & 825.92 & 822.23 & 1.01 & & \\
\hline$\nu_{8}$ & & 921.49 & & 939.86 & 934.42 & 12.92 & & \\
\hline$\nu_{7}$ & & 944.50 & & 948.77 & 949.51 & 5.01 & & \\
\hline$\nu_{4}$ & & 1025.14 & & 1025.58 & 1024.93 & -0.21 & & \\
\hline$\nu_{6}$ & & 1206.51 & & 1225.41 & 1224.09 & 17.58 & & \\
\hline$\nu_{3}$ & & 1328.31 & & 1343.31 & 1342.28 & 13.96 & & \\
\hline$\nu_{12}$ & $1436.65^{58}$ & 1434.90 & 1.75 & 1442.44 & 1440.68 & 5.78 & 5.79 & 0.01 \\
\hline$\nu_{2}$ & & 1583.97 & & 1626.17 & 1624.18 & 40.21 & & \\
\hline$\nu_{11}$ & $2969.60^{59}$ & 2966.46 & 3.14 & 2988.63 & 2985.35 & 18.88 & 19.03 & 0.15 \\
\hline$\nu_{1}$ & & 3008.22 & & 3021.85 & 3019.06 & 10.84 & & \\
\hline$\nu_{5}$ & & 3068.79 & & 3082.36 & 3079.78 & 10.98 & & \\
\hline$\nu_{9}$ & & 3090.52 & & 3104.33 & 3101.65 & 11.13 & & \\
\hline rms & & & 2.54 & & & & & \\
\hline
\end{tabular}

All values are in $\mathrm{cm}^{-1}$.

${ }^{a}$ Isotopic shifts calculated as $\Delta=E\left({ }^{12} \mathrm{C}_{2} \mathrm{H}_{4}\right)-E\left({ }^{13} \mathrm{C}_{2} \mathrm{H}_{4}\right)$.

Not surprisingly, the empirical optimization of the equilibrium geometry gives much smaller residual errors with respect to all pure ab initio predictions. These rotational levels up to $J=8$ for the ground state and $J=5$ for the $\nu_{9} / \nu_{11}$ band are given in Tables X and XI. A Comparison with experimental data analysis reported by Loroño et al. ${ }^{8}$ shows a very good agreement: the rms deviation being $0.00062 \mathrm{~cm}^{-1}$ for the ground state and $0.01 \mathrm{~cm}^{-1}$ for the $\nu_{9} / \nu_{11}$ band. Again, this confirms a considerable progress in the PES accuracy reported in the present work. Rotational study for isotopic species is under investigation.

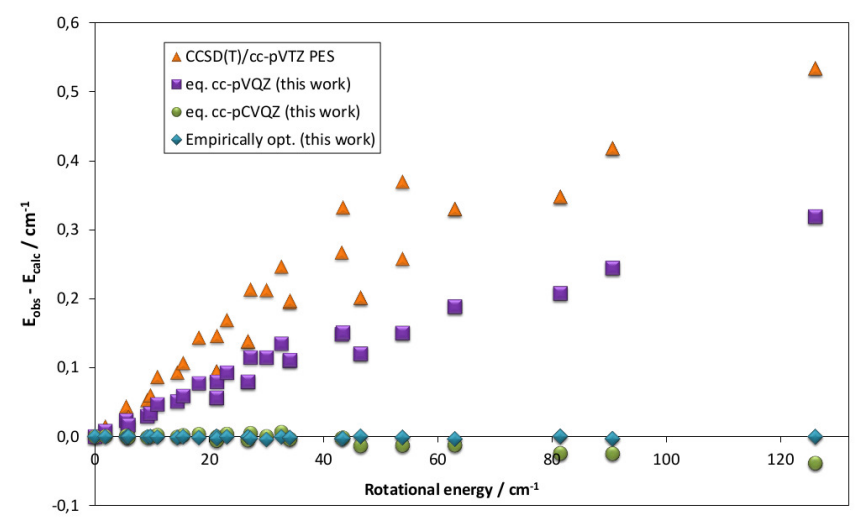

Figure 5. Observed minus calculated discrepancies of G.S. rotational levels for ${ }^{12} \mathrm{C}_{2} \mathrm{H}_{4}$ up to $J=5$ for three sets of equilibrium geometry parameters. Comparison with our calculation of rotational levels using the PES "morsified" by Avila and Carrington ${ }^{32}$ is included. "Empirically opt." stands for "Empirically optimized equilibrium".

\section{DISCUSSION AND SUMMARY}

Previous works on the vibrational and rovibrational theoretical predicitions and on spectra analyses for ethylene have shown the limitation of the CCSD(T)/cc-pVTZ PES and the need of a new more accurate PES at a higher level of $a b$ initio theory. The construction of such a 12D PES for this molecule represents a challenging issue. In this work we applied extended ab initio calculation on a dense grid of 82542 nuclear configurations at the CCSD(T)/cc-pVQZ level of the theory to determine the shape of electronic ground state PES more precisely involving higher-order terms. A fitting procedure of $a b$

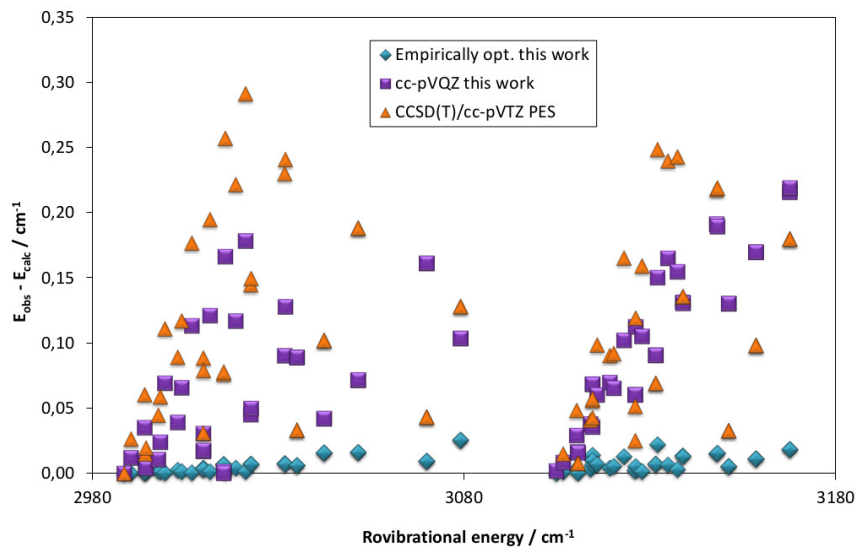

Figure 6. Rotational dependence of the Obs-Calc. errors for the $\nu_{9} / \nu_{11}$ bands of ${ }^{12} \mathrm{C}_{2} \mathrm{H}_{4}$ up to $J=5$ for two sets of equilibrium geometry parameters. cc-pVTZ results correspond to calculations made using Avila and Carrington $\mathrm{PES}^{32}$. In all calculations the pure vibrational error was substracted (see the text). "Empirically opt." stands for "Empirically optimized equilibrium". 
Table X. Rotational energy levels of the ground state of ${ }^{12} \mathrm{C}_{2} \mathrm{H}_{4}$ computed from our optimized PES compared to values deduced from experiment (Ref. 8).

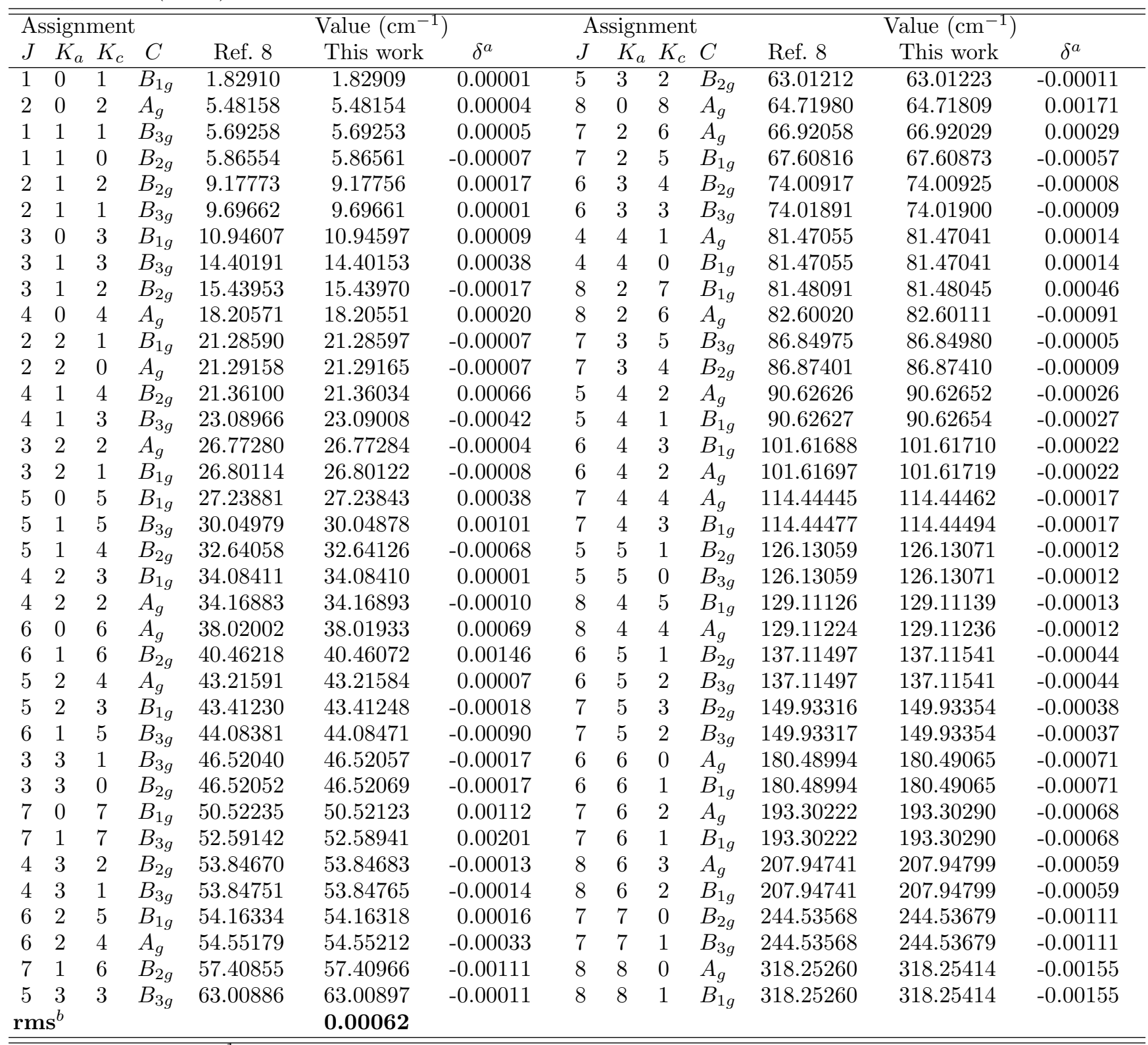

All values are in $\mathrm{cm}^{-1}$.

${ }^{a} \delta$ is the deviation from Ref. 8 ground state levels, deduced from experimental spectra.

${ }^{b}$ Root-mean-squares (Obs.-Calc.) deviation for ground state energy levels up to $J=8$.

initio electronic energies using a sixth PES expansion in curvilinear symmetry-adapted coordinates involving 2650 parameters were applied, with a total rms deviation of $1.37 \mathrm{~cm}^{-1}$. This allowed a considerable improvement of vibrational predictions compared to results presently available in the literature. Pure $a b$ initio variational fundamental band center predictions are now very close to experimental values with an average error below $3 \mathrm{~cm}^{-1}$. Nuclear motion calculations were carried out by a variational method accounting for all symmetry properties as well as relevant transformations. This approach was also applied for the vibrational levels calculation of two isotopic species of $D_{2 h}$ symmetry ${ }^{12} \mathrm{C}_{2} \mathrm{D}_{4}$ and ${ }^{13} \mathrm{C}_{2} \mathrm{H}_{4}$, with an error on fundamental band centers below $2.5 \mathrm{~cm}^{-1}$. For the rotational calculations, we evaluated the impact of changes in equilibrium nuclear geometrical parameters on the rotational levels. Test calculations were performed by keeping the shape of the potential function cc-pVQZ PES (because the full grid 12D calculations at higher levels of $a b$ initio theory are too demanding), but only 
Table XI. Rotational dependence of energy levels for the $\nu_{9} / \nu_{11}$ dyad of ${ }^{12} \mathrm{C}_{2} \mathrm{H}_{4}$ compared to values deduced from experimental spectra (Ref. 8).

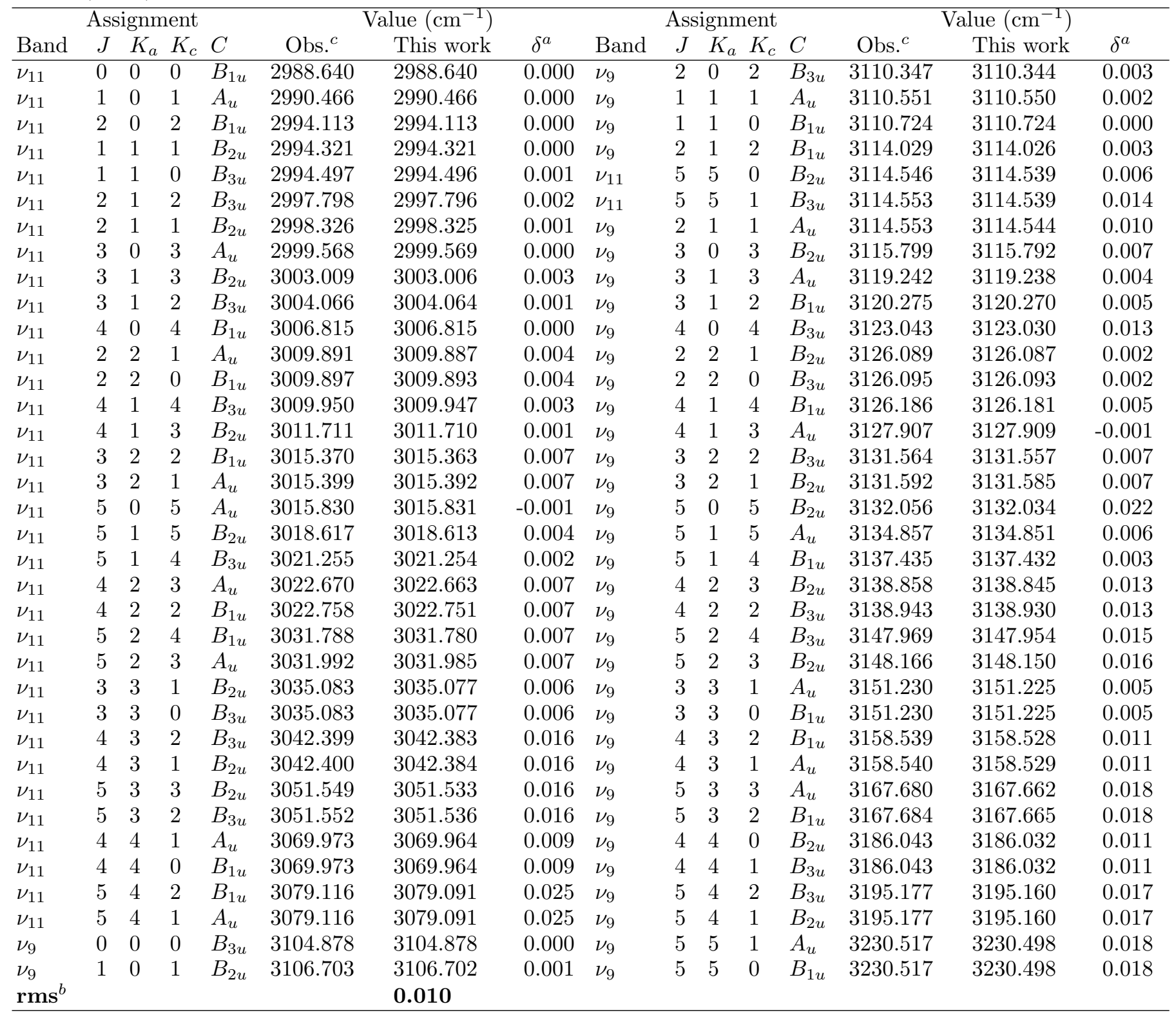

All values are in $\mathrm{cm}^{-1}$.

${ }^{a} \delta$ is the deviation from Ref. $8 \nu_{9} / \nu_{11}$ dyad energy levels, band centers being matched to experimental values in VSS procedure.

${ }^{b}$ Root-mean-squares (Obs.-Calc.) deviation for $\nu_{9} / \nu_{11}$ polyad up to $J=5$.

${ }^{c}$ Obs.: Levels deduced from experimental spectra, Ref. 8.

changing the equilibrium geometry. Empirical optimization of the equilibrium configuration procedure was necessary to reach the accuracy expected in high-resolution spectroscopy applications.

The final PES of the electronic ground state, constructed from the $a b$ initio $\operatorname{CCSD}(\mathrm{T}) / \mathrm{cc}-\mathrm{pVQZ}$ potential function, and combined with empirically optimized nuclear geometries gives considerably improved rovibrational levels. Up to $J=8$ the $\operatorname{RMS}(O b s .-$ Calc.) deviation was below $0.0007 \mathrm{~cm}^{-1}$ for ground state rotational levels of ${ }^{12} \mathrm{C}_{2} \mathrm{H}_{4}$. A quite smooth rotational trend for the errors was also obtained for rovibrational bands, with a RMS(Obs. - Calc. $)$ deviation of $0.01 \mathrm{~cm}^{-1}$ up to $J=5$ for the $\nu_{9} / \nu_{11}$ dyad after the matching of band centers to experimental values . An extension of this work for rovibrational spectra of isotopic species of $D_{2 h}$ symmetry ${ }^{12} \mathrm{C}_{2} \mathrm{D}_{4}$ and ${ }^{13} \mathrm{C}_{2} \mathrm{H}_{4}$ is planned, as well as for symmetry breaking isotopologues ${ }^{12} \mathrm{C}_{2} \mathrm{H}_{3} \mathrm{D},{ }^{12} \mathrm{C}_{2} \mathrm{D}_{3} \mathrm{H}$ 
and ${ }^{12} \mathrm{C}_{2} \mathrm{H}_{2} \mathrm{D}_{2}$. In order to improve the accuracy of fundamentals and first overtones band centers, empirical quadratic corrections to the PES are currently under investigation. We provide the CCSD $(\mathrm{T}) / \mathrm{cc}-\mathrm{pVQZ}$ PES, presented in the symmetry-adapted coordinates defined in Eq. (3), as the electronic supplementary material ${ }^{67}$ of this paper. Expressions of the symmetry-adapted coordinates $S_{i}$ in terms of Cartesian coordinates are also provided in order to properly transform the PES. As an example, we give the third order PES expansion expressed in mass-weighted Cartesian displacements. Note that this latter form should not be considered for practical calculations.

As a next step we plan to derive effective spectroscopic models for vibration-rotation polyads from this PES by high-order contact transformation method as was recently reported for the methane molecule ${ }^{68}$. This would help a better description of complicated resonance perturbations for spectra analyses at extended wavenumber ranges. We also plan to explore other various basis sets as Morse, TPT, Kratzer, etc. in the future work.

\section{ACKNOWLEDGMENTS}

The support of Groupement de Recherche International SAMIA between CNRS (France), RFBR (Russia) and CAS (China) and Balaton program (France Hungary) is acknowledged. We acknowledge the support from IDRIS computer centre of CNRS France, Centre Informatique National de 1' Enseignement Supérieur (CINES) computer centre and of the computer centre Reims-Champagne-Ardenne. This work was granted access to the HPC resources of CINES/IDRIS under the allocation 2013-080538 made by GENCI. A.N. thanks the University of Reims for the invitation to work on this project. A support by Tomsk State University Competitiveness Improvement Program is acknowledged.

\section{REFERENCES}

${ }^{1}$ G. Tinetti, T. Encrenaz, and A. Coustenis, "Spectroscopy of planetary atmospheres in our Galaxy," Astronomy and Astrophysics Review 21, 63 (2013).

${ }^{2}$ C. de Bergh, R. Courtin, B. Bezard, A. Coustenis, E. Lellouch, M. Hirtzig, P. Rannou, P. Drossart, A. Campargue, S. Kassi, L. Wang, V. Boudon, A. Nikitin, and V. Tyuterev, Planetary And Space Science 61, 85 (2012).

${ }^{3}$ M. Rey, A. V. Nikitin, and V. G. Tyuterev, The Astrophysical Journal 789, 2 (2014).

${ }^{4}$ L. S. Rothman, I. E. Gordon, Y. Babikov, A. Barbe, D. C. Benner, P. F. Bernath, M. Birk, L. Bizzocchi, et al., Journal of Quantitative Spectroscopy \& Radiative Transfer 130, 4 (2013).

${ }^{5}$ V. Tyuterev, Y. Babikov, S. Tashkun, V. Perevalov, A. Nikitin, J. Champion, C. Wenger, C. Pierre, G. Pierre, J. Hilico, and M. Loëte, Journal of Quantitative Spectroscopy \& Radiative Transfer 52, 459 (1994).

${ }^{6}$ Y. A. Ba, C. Wenger, R. Surleau, V. Boudon, M. Rotger, L. Daumont, D. A. Bonhommeau, V. G. Tyuterev, and M.-L. Dubernet,
Journal of Quantitative Spectroscopy \& Radiative Transfer $\mathbf{1 3 0}$ $62(2013)$.

${ }^{7}$ O. Ulenikov, O. Gromova, Y. Aslapovskaya, and V.-M. Horneman, Journal of Quantitative Spectroscopy \& Radiative Transfer 118, 14 (2013)

${ }^{8}$ M. A. Loroño Gonzalez, V. Boudon, M. Loëte, M. Rotger, M.T. Bourgeois, K. Didriche, M. Herman, V. Kapitanov, Y. Ponomarev, A. Solodov, A. Solodov, and T. Petrova, Journal of Quantitative Spectroscopy \& Radiative Transfer 111, 2265 (2010).

${ }^{9}$ T. Tan, S. Lau, P. Ong, K. Goh, and H. Teo, Journal of molecular spectroscopy 203, 310 (2000).

${ }^{10} \mathrm{G}$. Lebron and T. Tan, Journal of Molecular Spectroscopy 288, 11 (2013).

${ }^{11}$ M. Rotger, V. Boudon, and J. V. Auwera, Journal of Quantitative Spectroscopy \& Radiative Transfer 109, 952 (2008).

${ }^{12}$ O. Ulenikov, G. Onopenko, E. Bekhtereva, T. Petrova, A. Solodov, and A. Solodov, Molecular Physics 108, 637 (2010).

${ }^{13}$ H. Partridge and D. Schwenke, Journal of Chemical Physics 106, 4618 (1997)

${ }^{14} \mathrm{~T}$. Cours, P. Rosmus, and V. Tyuterev, Journal of Chemical Physics 117, 5192 (2002).

${ }^{15}$ O. Polyansky, A. Császár, S. Shirin, N. Zobov, P. Barletta, J. Tennyson, D. Schwenke, and P. Knowles, Science 299, 539 (2003).

${ }^{16}$ V. G. Tyuterev, L. Rgalia-Jarlot, D. W. Schwenke, S. A. Tashkun, and Y. G. Borkov, Comptes Rendus Physique 5, 189 (2004), gas phase molecular spectroscopy.

${ }^{17}$ L. Lodi, R. N. Tolchenov, J. Tennyson, A. E. Lynas-Gray, S. V. Shirin, N. F. Zobov, O. L. Polyansky, A. G. Császár, J. N. P. van Stralen, and L. Visscher, Journal of Chemical Physics 128, 044304 (2008).

${ }^{18}$ A. Nikitin, F. Holka, V. Tyuterev, and J. Fremont, Journal of Chemical Physics 130, 244312 (2009).

${ }^{19}$ S. Yurchenko, J. Zheng, H. Lin, P. Jensen, and W. Thiel, Journal of Chemical Physics 123, 134308 (2005).

${ }^{20}$ X. Huang, D. W. Schwenke, and T. J. Lee, Journal of Physical Chemistry A 113, 11954 (2009).

${ }^{21}$ S. Carter, A. R. Sharma, J. M. Bowman, P. Rosmus, and R. Tarroni, Journal of Chemical Physics 131, 224106 (2009).

${ }^{22}$ P. Barletta, S. V. Shirin, N. F. Zobov, O. L. Polyansky, J. Tennyson, E. F. Valeev, and A. G. Császár, Journal of Chemical Physics 125, 204307 (2006).

${ }^{23}$ P. G. Szalay, F. Holka, J. Fremont, M. Rey, K. A. Peterson, and V. G. Tyuterev, Physical Chemistry Chemical Physics 13, 3654 (2011).

${ }^{24}$ F. Holka, P. G. Szalay, J. Fremont, M. Rey, K. A. Peterson, and V. G. Tyuterev, Journal of Chemical Physics 134, 094306 (2011).

${ }^{25}$ M. Pavanello, L. Adamowicz, A. Alijah, N. F. Zobov, I. I. Mizus, O. L. Polyansky, J. Tennyson, T. Szidarovszky, and A. G. Csaszar, Journal of Chemical Physics 136, 184303 (2012).

${ }^{26}$ A. V. Nikitin, M. Rey, and V. G. Tyuterev, Chemical Physics Letters 501, 179 (2011).

${ }^{27}$ X. Huang, D. W. Schwenke, and T. J. Lee, Journal of Chemical Physics 134, 044320 (2011).

${ }^{28}$ M. Rey, A. V. Nikitin, and V. G. Tyuterev, Phys. Chem. Chem. Phys. 15, 10049 (2013).

${ }^{29}$ V. G. Tyuterev, R. V. Kochanov, S. A. Tashkun, F. Holka, and P. G. Szalay, Journal of Chemical Physics 139, 134307 (2013).

${ }^{30}$ D. Strobusch and C. Scheurer, Journal of Chemical Physics 140, 074111 (2014).

${ }^{31}$ A. V. Nikitin, X. Thomas, L. Regalia, L. Daumont, P. Von der Heyden, V. I. G. Tyuterev, L. Wang, S. Kassi, and A. Campargue, Journal of Quantitative Spectroscopy \& Radiative Transfer 112, 28 (2011).

${ }^{32} \mathrm{G}$. Avila and T. Carrington, The Journal of Chemical Physics 135, 064101 (2011).

${ }^{33}$ J. M. L. Martin, T. J. Lee, P. R. Taylor, and J. Franois, The Journal of Chemical Physics 103, 2589 (1995).

${ }^{34}$ S. Carter, A. R. Sharma, and J. M. Bowman, The Journal of Chemical Physics 137, 154301 (2012). 
${ }^{35}$ J. Watson, Molecular Physics 15, 479 (1968).

${ }^{36}$ G. D. Purvis and R. J. Bartlett, The Journal of Chemical Physics 76, 1910 (1982).

${ }^{37}$ K. Raghavachari, G. W. Trucks, M. Head-Gordon, and J. A. Pople, Chemical Physics Letters 157, 479 (1989).

${ }^{38}$ T. H. Dunning, The Journal of Chemical Physics 90, 1007 (1989).

${ }^{39}$ H.-J. Werner, P. J. Knowles, G. Knizia, F. R. Manby, M. Schütz, et al., "Molpro, version 2012.1, a package of ab initio programs," (2012), see https://www.molpro.net/.

${ }^{40}$ F. Pawlowski, P. Jorgensen, J. Olsen, F. Hegelund, T. Helgaker, J. Gauss, K. Bak, and J. Stanton, Journal of Chemical Physics 116, 6482 (2002).

${ }^{41}$ J. Duncan, D. McKean, and P. Mallinson, Journal of Molecular Spectroscopy 45, 221 (1973).

${ }^{42}$ J. Duncan and E. Hamilton, Journal of Molecular Structure 76, 65 (1981).

${ }^{43}$ A. Nikitin, J. Champion, and V. Tyuterev, Journal of Molecular Spectroscopy 182, 72 (1997).

${ }^{44}$ D. Schwenke and H. Partridge, Spectrochimica Acta - Part A: Molecular and Biomolecular Spectroscopy 57, 887 (2001).

${ }^{45}$ M. Rey, A. V. Nikitin, and V. G. Tyuterev, Journal of Chemical Physics 136, 244106 (2012).

${ }^{46}$ P. Jensen, Journal of Molecular Spectroscopy 128, 478 (1988).

${ }^{47}$ J. Tennyson, J. Henderson, and N. Fulton, Computer Physics Communications 86, 175 (1995).

${ }^{48}$ C. Fábri, E. Mátyus, T. Furtenbacher, L. Nemes, B. Mihály, T. Zoltáni, and A. G. Császár, The Journal of Chemical Physics 135, 094307 (2011).

${ }^{49}$ A. G. Császar, C. Fábri, T. Szidarovszky, E. Mátyus, T. Furtenbacher, and G. Czakó, Phys. Chem. Chem. Phys. 14, 1085 (2012).

${ }^{50}$ M. Rey, A. V. Nikitin, and V. G. Tyuterev, Molecular Physics 108, 2121 (2010).

${ }^{51}$ R. Georges, M. Bach, and M. Herman, Molecular Physics 97, 279 (1999).

${ }^{52}$ A.-K. Mose, F. Hegelund, and F. Nicolaisen, Journal of Molec- ular Spectroscopy 137, 286 (1989).

${ }^{53}$ F. Mompeán, R. Escribano, S. Montero, J. Bendtsen, and R. Butcher, Journal of Molecular Spectroscopy 116, 48 (1986).

${ }^{54}$ T. L. Tan, M. G. Gabona, and G. B. Lebron, Journal of Molecular Spectroscopy 266, 113 (2011).

${ }^{55}$ T. Tan, K. Goh, P. Ong, and H. Teo, Journal of molecular spectroscopy 202, 249 (2000).

${ }^{56}$ J. Duncan, Molecular Physics 83, 159 (1994).

${ }^{57}$ J. Duncan and A. Ferguson, The Journal of Chemical Physics 89, 4216 (1988).

${ }^{58}$ T. Tan, K. Goh, P. Ong, and H. Teo, Journal of Molecular Spectroscopy 207, 189 (2001).

${ }^{59}$ T. Tan, L. Kang, K. Goh, and H. Teo, Chemical Physics Letters 393, 343 (2004).

${ }^{60}$ J. Harper, A. Morrison, and J. Duncan, Chemical Physics Letters 83, 32 (1981).

${ }^{61}$ T. Tan, K. Goh, P. Ong, and H. Teo, Chemical Physics Letters 315, 82 (1999).

${ }^{62}$ K. Goh, T. Tan, P. Ong, and H. Teo, Molecular Physics 98, 583 (2000).

${ }^{63}$ A. V. Nikitin, Journal of Molecular Spectroscopy 252, 17 (2008).

${ }^{64}$ W. Raballand, M. Rotger, V. Boudon, and M. Loëte, Journal of Molecular Spectroscopy 217, 239 (2003).

${ }^{65}$ D. Papousek and M. R. Aliev, Molecular Vibrational-Rotational Spectra (Elsevier Scientific, New York, 1982).

${ }^{66} \mathrm{~V}$. Boudon, M. Rotger, N. Zvereva-Loëte, and M. Loëte, Journal of Molecular Structure 780781, 124 (2006).

${ }^{67}$ See supplemental material at [URL will be inserted by AIP] for the parameters of our sixth order PES presented in symmetryadapted coordinates (appendix1). We also provide the expressions of the symmetry-adapted coordinates in terms of Cartesian ones (appendix2). As an example, the third order PES expansion in terms of mass-weighted Cartesian displacements is given (appendix3).

${ }^{68}$ V. Tyuterev, S. Tashkun, M. Rey, R. Kochanov, A. Nikitin, and T. Delahaye, Journal of Physical Chemistry A 117, 13779 (2013). 